\title{
Extensive stage-regulation of translation revealed by ribosome profiling of Trypanosoma brucei
}

\author{
Bryan C Jensen ${ }^{1 \dagger}$, Gowthaman Ramasamy ${ }^{1 \dagger}$, Elton J R Vasconcelos ${ }^{1}$, Nicholas T Ingolia ${ }^{2}$, Peter J Myler ${ }^{1,3,4}$ \\ and Marilyn Parsons 1,3* $^{*}$
}

\begin{abstract}
Background: Trypanosoma brucei subspecies infect humans and animals in sub-Saharan Africa. This early diverging eukaryote shows many novel features in basic biological processes, including the use of polycistronic transcription to generate all protein-coding mRNAs. Therefore we hypothesized that translational control provides a means to tune gene expression during parasite development in mammalian and fly hosts.
\end{abstract}

Results: We used ribosome profiling to examine genome-wide protein synthesis in animal-derived slender bloodstream forms and cultured procyclic (insect midgut) forms. About one-third of all CDSs showed statistically significant regulation of protein production between the two stages. Of these, more than two-thirds showed a change in translation efficiency, but few appeared to be controlled by this alone. Ribosomal proteins were translated poorly, especially in animal-derived parasites. A disproportionate number of metabolic enzymes were up-regulated at the mRNA level in procyclic forms, as were variant surface glycoproteins in bloodstream forms. Comparison with cultured bloodstream forms from another strain revealed stage-specific changes in gene expression that transcend strain and growth conditions. Genes with upstream ORFs had lower mean translation efficiency, but no evidence was found for involvement of uORFs in stage-regulation.

Conclusions: Ribosome profiling revealed that differences in the production of specific proteins in T. brucei bloodstream and procyclic forms are more extensive than predicted by analysis of mRNA abundance. While in vivo and in vitro derived bloodstream forms from different strains are more similar to one another than to procyclic forms, they showed many differences at both the mRNA and protein production level.

Keywords: Ribosomal proteins, Ribosome profiling, Stage-regulation, Trypanosome, Translation

\section{Background}

Most organisms exhibit robust gene regulation at the level of transcription. Among the exceptions to this rule are the trypanosomatid parasites, including Trypanosoma brucei. Nonetheless, as T. brucei transits its life cycle through the mammalian and insect hosts, large changes in protein expression occur [1-3]. Whilst previous microarray and RNA-seq studies [4-12] have shown that a moderate number of transcripts are developmentally regulated, primarily as a result of differential mRNA stability

\footnotetext{
* Correspondence: mparsons@u.washington.edu

${ }^{\dagger}$ Equal contributors

'Seattle Biomedical Research Institute, 307 Westlake Ave N, Seattle, WA 98109-5219, USA

${ }^{3}$ Department of Global Health, University of Washington, Harris Hydraulics Building, 1705 NE Pacific St \#310E, Box 357965, Seattle, WA 98195, USA Full list of author information is available at the end of the article
}

[13-15], much less is known about the role of translational regulation. A limited number of individual genes have been shown to be developmentally regulated at the level of translation [15-18] and numerous examples of discrepancies between stage-specific changes in mRNA and protein level exist. A recent study has noted some changes in the association of mRNAs with polysomes in developing mammalian bloodstream forms (BF) [19] and initial work suggests that the changes in translation efficiency occur between cultured BF and cultured insect stages [20]. The work presented here aimed to define the role of translational control in modulating differences in gene expression during parasite development.

Trypanosoma brucei spp. are the causative agents of lethal human African trypanosomiasis (African sleeping sickness) and nagana, a wasting disease in cattle. The presence of T. brucei and related African trypanosomes 
in sub-Saharan Africa has had a major impact on development, affecting humans directly as well as indirectly through impact on livestock. African trypanosomes share molecular mechanisms of gene regulation with the agents of Chagas' disease (Trypanosoma cruzi) and leishmaniasis (Leishmania spp.). The most striking feature of nuclear gene expression in these organisms is the organization of genes into long polycistronic clusters, such that individual genes lack promoters [21-23]. The polycistronic mRNAs are processed into individual transcripts by trans-splicing of a common capped mini-exon sequence (the spliced leader, SL) to the downstream coding sequence (CDS) and concomitant polyadenylation of the upstream gene [24]. Thus all mRNAs bear the same sequence at their $5^{\prime}$ terminus, upstream of the gene-specific untranslated region. Despite the ubiquity of trans-splicing, cis-splicing is extremely rare, with only two known examples in $T$. brucei. Interestingly, mRNAs derived from the same polycistronic cluster are not generally expressed to similar levels, nor do they tend to show the same patterns of developmental regulation. These differences in mRNA abundance are thought to be mediated post-transcriptionally, in large part by differential stability resulting from interactions with RNA binding proteins $[13,25]$.

The extent of gene regulation at the mRNA level is a major contributor to differential protein expression in most species. However, additional levels of regulation are known to yield different levels of expression of various proteins under a given condition as well as modulating how those levels change upon perturbation. For example, a recent study that dissected the contributions of level of transcription, mRNA turnover, translation, and protein degradation demonstrated that translational efficiency was the largest contributor to predicting protein abundance across genes [26]. Factors that contribute to translational efficiency include gene-specific features, such as the context of the start codon, the presence of upstream open reading frames ( $\mathrm{uORFs}$ ), the length and sequence composition of the $5^{\prime}$ UTR, and the presence of protein binding sites in the untranslated regions (UTRs) [27-29]. Changes in the cellular milieu can also affect translation by modulating abundance or modification of translation factors (e.g., phosphorylation of eIF $2 \alpha$ ), altering the abundance of different RNA binding proteins or microRNAs, perturbing protein folding and changing polyadenylation $[29,30]$. Other studies have shown that translational controls play a prominent role in oncogenesis of mammalian cells [31], induction of the unfolded protein response in Toxoplasma gondii [32], exposure to light in Arabidopsis [33], and during development in Plasmodium [34,35], to name a few. In trypanosomatids, given the lack of transcriptional controls, we hypothesized that translational regulation would play a prominent role in parasite development.

To directly test this hypothesis, we made use of the recently developed technique of ribosome profiling [36,37], which quantitatively interrogates the positions of all ribosomes on their mRNA templates, thus providing a comprehensive picture of cellular translation. Our results show extensive changes in gene-level protein production between in vivo-derived slender BF (slBF) and insect midgut stages (procyclic cultured forms, PCF), greater in extent and magnitude than changes in mRNA abundance. Of the 8398 intact genes studied, thousands of genes show changes in protein synthesis mediated by both mRNA abundance and translational efficiency, but less than 200 genes appear to be regulated by changes in translation efficiency. Comparison with cultured BF (cBF) from another strain allowed the further definition of changes in protein production associated with growth conditions and strain variation.

\section{Results and discussion}

The total protein synthetic activity devoted to a given gene is determined by both its mRNA abundance and the efficiency with which its mRNA is translated. To assess the extent of translational regulation during $T$. brucei development we pursued a genome-wide ribosome profiling approach [36]. Our primary focus was on two rapidly proliferating life cycle stages (PCF and slBF) that are readily available for the pleiomorphic T. brucei strain 927, which has retained the ability to differentiate and also has the most complete genome sequence available. We also examined cultured $\mathrm{BF}$ (cBF) from another often-used strain, T. brucei 427 (see Additional file 1: Table S1 for sample description). Figure 1A shows an overview of the workflow, with libraries being prepared and sequenced from three biological replicates of each condition. This approach relies on the ability of the translating ribosome to protect a footprint of $\sim 28 \mathrm{nt}$ from RNase digestion (see Additional file 2: Figure S1). Ribosome-protected fragments are purified and used to generate libraries for high throughput sequencing. The read counts from these libraries reflect the extent of translation of each gene, allowing quantitative measurement of gene expression between samples. Moreover, comparison with the read counts from fragmented poly(A) + mRNA libraries prepared using the same biological samples reveals the relative contributions of changes in mRNA abundance and translational efficiency to regulation of protein production. We also constructed libraries that specifically captured the $5^{\prime}$ ends of the mRNAs using SL RNA-seq (see Methods), assisting in refinement of the annotated CDSs.

Reads were mapped to the T. brucei 927 genome and assigned to individual genes (as described in Methods), 


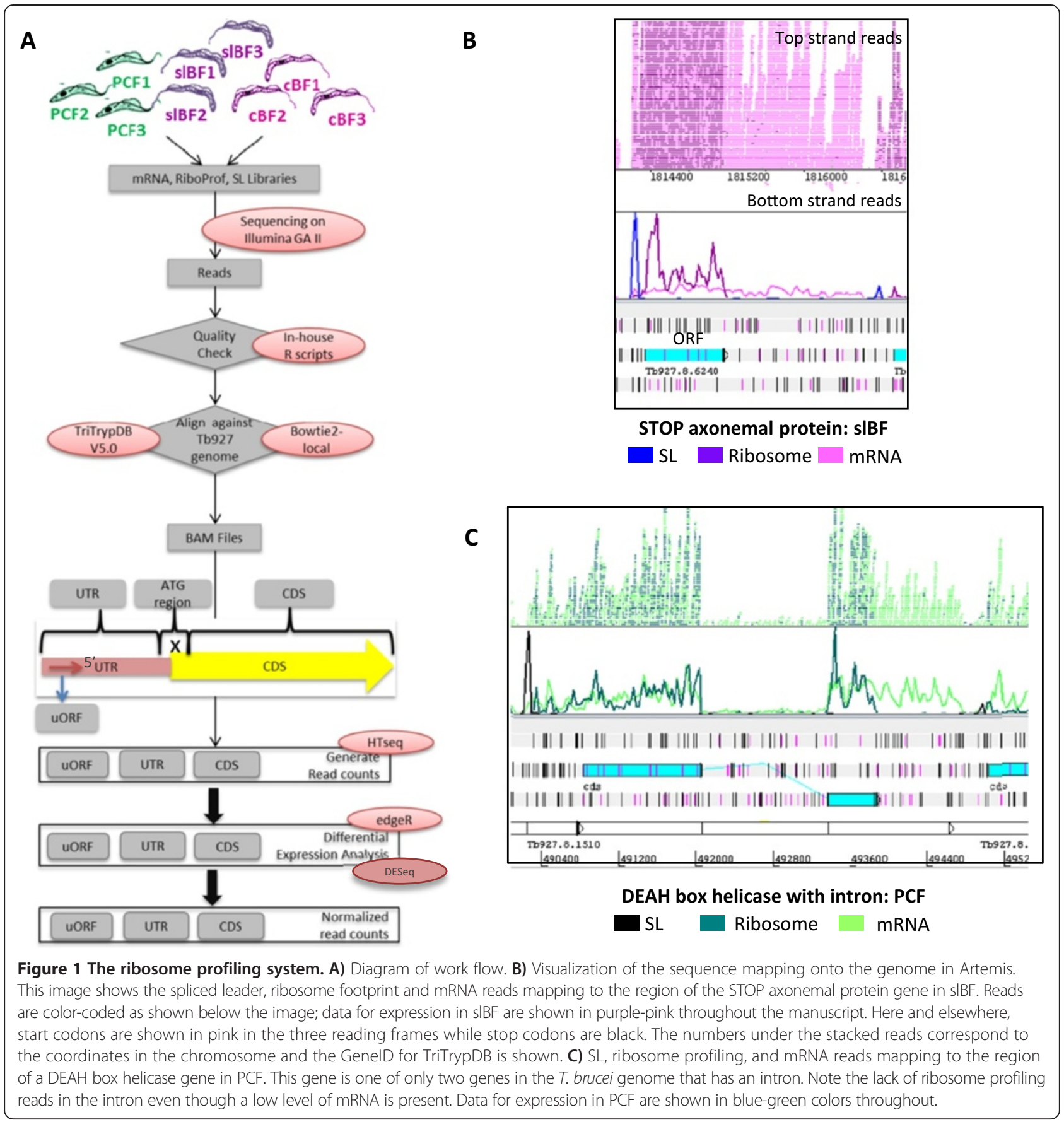

yielding 6-15 million uniquely mapping reads per biological sample (see Additional file 1: Table S2 for statistics). Additional file 3 provides gene-level read count data for all 9141 annotated CDSs, newly identified CDSs, and pseudogenes. The ribosome footprints showed the characteristic 3 nucleotide periodicity, being enriched for reads starting at the first nucleotide of each codon (Additional file 2: Figure S1c), while mRNAs reads were relatively evenly distributed across the nucleotide positions. As expected, mRNA reads extend from the site of the SL through the CDS and terminate at the most 3' polyA site, usually $\sim 100 \mathrm{nt}$ upstream of the predominant $\mathrm{SL}$ site of the downstream gene. In contrast, ribosome footprint reads span the CDS from 12 nt prior to start codon to 9 nt past the stop codon and are absent from the 3' untranslated region (UTR) (see Additional file 1: Table S3 and Additional file 2: Figure S4). Ribosome release scores (RRSs, also referred to as the disengagement score), a metric of translation that compares the density of ribosome footprints on the CDS to that in the 3' UTR, using 
mRNA as a control, were calculated for each intact CDS (except the $\sim 8 \%$ for which read counts were very low, see Methods) [38,39]. Of those genes for which scores could be calculated, $87 \%$ had scores $>10$ and $60 \%$ had scores $>50$, indicating most mRNAs had considerable enrichment for ribosome footprints in the CDS (see Additional file 3). Technical replicates showed high reproducibility in read counts for both ribosome footprints and mRNA, whereas biological replicates showed more variation (Additional file 2: Figure S2A). Nonetheless, correlation coefficients between gene-level read counts for biological replicates were high for both ribosome footprints $\left(r^{2}=0.86-0.94\right)$ and mRNA $\left(r^{2}=0.74-0.94\right)$ (Additional file 2: Figure S3). Both ribosome footprint and mRNA reads were highly strand-specific (see Figure 1B), but the distributions of edgeR [40] normalized read counts per gene were noticeably different between ribosome footprints and mRNA (Additional file 2: Figure S2B), with the ribosome footprint read counts showing a broader spread than the mRNA reads. Only two T. brucei genes undergo both cis and trans-splicing. For these genes, ribosome footprint reads are abundant in the exons, but absent in the intron, whereas there are readily detectable mRNA reads in the latter (see Figure 1C).

To assess whether RNA binding proteins might protect the portion of the mRNA with which they interact to yield similarly sized fragments as ribosome protection (and that RNA-protein complex would sediment under the conditions used to pellet the ribosome), we examined several transcripts with 3' UTRs known to bind specific proteins. No significant ribosome footprint peaks were observed within the 3' UTRs of the GPEET2, ZC3H11 and PGKB mRNAs (see Additional file 2: Figure S5), despite extensive evidence that they bind multiple different proteins [41-43]. As seen in Figure 1B, there was often a ribosome footprint peak close to the CDS start codon (see Additional file 2: Figure S4), possibly due to an artifact of cycloheximide treatment [36], which blocks elongation but not initiation. For this reason, the first 45 nt of the CDS were not included in the gene-level read counts (see Methods). Although T. brucei 5' UTRs are generally short (the median from our SL data being $87 \mathrm{nt}$, not including the SL itself) many of the longer 5' UTRs clearly show ribosome footprints that are distinct from the peak at the start codon (Figure 1C). The median ribosome footprint read density in the $5^{\prime}$ UTRs correlated with those of the corresponding CDSs $\left(R^{2}=0.43-0.62\right.$, depending on the condition), but was generally lower (Additional file 1: Table S3). Recent work has demonstrated that ribosome footprints on noncoding RNAs and noncoding regions of mRNAs (such as the $5^{\prime}$ UTR) can be discriminated from translation of functional proteincoding genes because the profile does not terminate at stop codons, likely due to the presence of weak translation in multiple overlapping reading frames [38,39]. For most genes in our study, these 5' UTR footprints were not associated with any ORFs starting with an ATG, and they continued through the 5' UTR irrespective of the presence of stop codons; hence in most cases they do not represent specifically translated upstream ORFs (uORFs). These protected fragments may represent assembled $80 \mathrm{~S}$ ribosomes, as suggested from similar observations in yeast and mammalian cells [44] or possibly protection by the scanning complex.

\section{General aspects of the translational landscape}

Ribosome profiling provides a comprehensive overview of the genes to which cells devote the most translational resources, which reflects a far greater biosynthetic commitment than mRNA production. The mRNA reads and ribosome footprint reads were plotted for each CDS (expressed as edgeR-normalized reads/kb, RPK) for each biological sample (color-coded in Figure 2A). Several features are immediately apparent. While the two parameters are positively correlated (with $\mathrm{R}^{2} \sim 0.7$ ), the relationship is not strictly linear. Indeed, mRNAs expressed to similar levels can show a large variation in their association with ribosomes. For example, CDSs with mRNA read counts from 500-700 RPK in PCF sample 2 had corresponding ribosome footprint reads ranging from $<10$ to $>6000$ (see Figure $2 \mathrm{~B}$ ). Thus, the translational efficiency (TE, calculated as the ratio of ribosome footprint read counts to mRNA read counts for each CDS) of mRNAs varies dramatically even within this narrow range of transcript abundance. In order to compare the sensitivity of ribosome profiling with standard polysome analysis, we examined four single-copy genes with varying TE, but similar mRNA length and abundance in PCF. Northern analysis revealed the distribution of these mRNAs between differently sized polysomes separated by sucrose gradient fractionation of PCF lysates (Additional file 2: Figure S6). Ribosome profiling indicates that Tb927.9.8740 (which encodes the RNA binding protein DRBD3) has a TE of 3.74 in PCF, placing it at the $99^{\text {th }}$ percentile for that stage (see Additional file 2: Figure S2C); while Tb927.1.4690 (which encodes the protein arginine methyl transferase PRMT1) has a TE of 1.10. Both mRNAs were associated with higher-order polysomes, although the four-fold difference in TE is barely detectable on the gradient fractionation due to compression of larger polysomes at the bottom of the gradient. In contrast, Tb927.9.4360 (which encodes the kinetoplast RNA editing ligase KREL1), has a much lower TE (0.29) and peaked in the monosome fraction, although a small amount of association with larger polysomes was seen. Similar results (not shown) were seen for Tb927.8.2650 (which encodes a putative metallo- $\beta$-lactamase-like protein), with a TE 

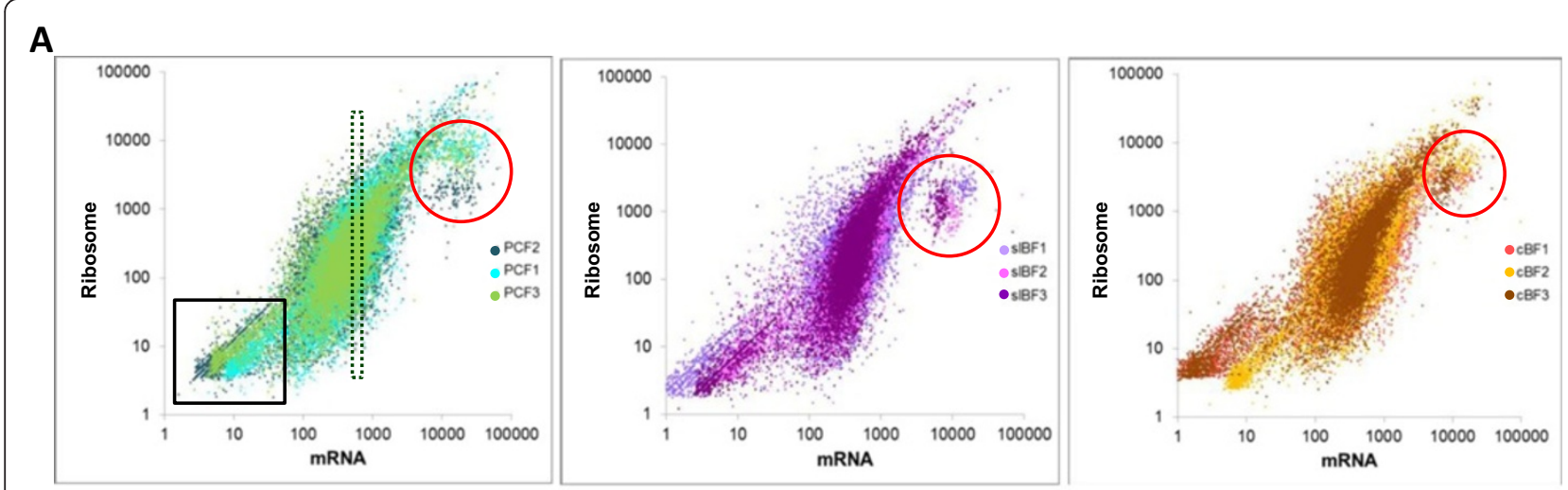

B

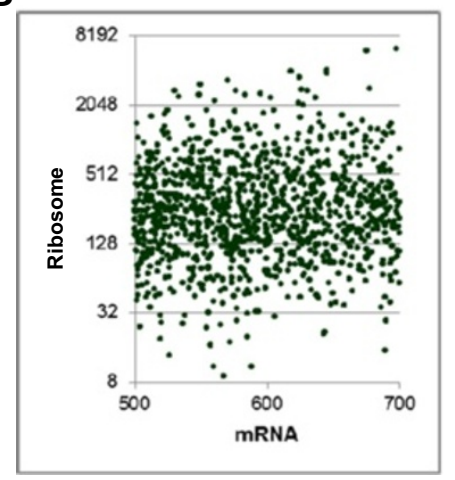

C

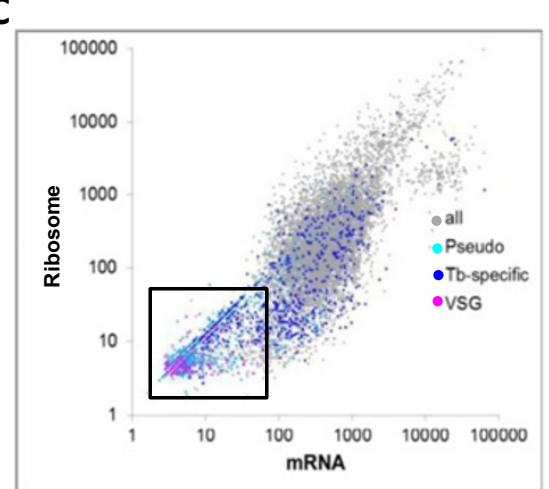

D

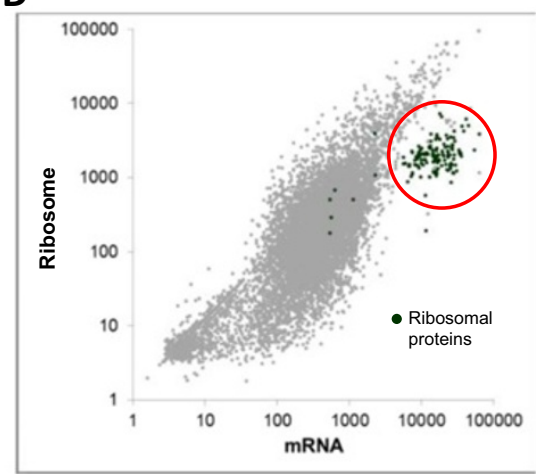

Figure 2 Overview of the translational landscape. A) Ribosome footprint and mRNA edgeR-normalized RPK for all genes, including pseudogenes, are shown. Each panel includes all biological replicates for a given stage, which are shown in different shades. The box outlines the genes with $<50$ RPK, the dotted box is enlarged in panel B; and the circle marks a set of genes with high mRNA read counts but relatively lower ribosome read counts that is referred to in the text. B) Illustration of large differences in ribosome association with mRNAs expressed to similar levels in PCF sample 2. Note that the $x$-axis is linear and the $y$-axis is $\log _{2}$. C) Expression levels of pseudogenes, VSG genes and T. brucei specific genes in PCF. The boxed area (<50 RPK) is comprised mostly of pseudogenes (cyan dots) and VSG genes (pink dots) and a subset of the T. brucei specific genes (blue dots). D) The cluster of genes with reduced translation efficiency corresponds to structural components of the cytosolic ribosome (green dots).

of 0.09. Thus, as well as providing TE data for essentially all genes, ribosome profiling is more quantitative and has a greater dynamic range than traditional polysome analysis.

It is apparent from Figure 2A that there are two clusters of genes with relationships between translation and mRNA abundance that are distinct from the majority. One group (indicated by the black box) likely represents genes that are expressed at only very low levels, if at all, since both their ribosome footprint and mRNA RPK were $<50$. As expected, a large proportion of pseudogenes and variant surface glycoprotein (VSG) genes, which are expressed clonally during antigenic variation in BF and not expressed in PCF, fall in this sector (Figure 2C). While many genes encoding T. bruceispecific hypothetical proteins also have low ribosome footprint read counts, the majority show higher levels of expression.

The other cluster (indicated by the red circles in Figure 2A) is composed of mRNAs with comparatively low TEs, despite having high mRNA expression levels. We observed that this cluster was comprised almost exclusively of genes corresponding to structural components of the cytoplasmic ribosome (Figure 2D, green dots). While this cluster was always separate from the main set of genes, the displacement varied between samples, both within and between stages. A few other proteins that are not known to be structural components of the ribosome lie within the cluster shown in Figure 2D. These include three genes (Tb927.9.8100, Tb927.9.8130, and Tb927.11.9700) encoding subunits of the nascent polypeptide associated complex, which associates with the ribosome and assists in protein folding [45], and one isoform of eukaryotic initiation factor 5a (Tb927.11.740). Also present are two newly identified genes described as ubiquitin fusion proteins (NTCDS TB.11.NT.154 and 155) [10]. These ubiquitin domains are fused to an RPL40 domain to generate RPL40, a protein of unknown function in the $60 \mathrm{~S}$ subunit. However, a similar ubiquitin fusion RPS31 is required for the 
functional integrity of eukaryotic 40S subunits [46]. Another three genes (NTCDS.TB.8.NT.93, 94, and 95) encode identical 34 amino acid proteins that show no conserved domains or sequence similarity, except to closely related species Trypanosoma vivax and Trypanosoma congolense. We speculate that the functions of these novel proteins may be related to the cytoplasmic ribosome.

\section{Changes in translation between stages}

High level unsupervised clustering of gene level ribosome footprint read counts from all nine samples (three sets of biological replicates of strain 927 PCF and in vivo-derived slBF, plus strain $427 \mathrm{cBF}$ ) was performed using edgeR. The resulting multidimensional scaling plot showed they fell into three distinct groups, with all BF samples separated from PCF samples by the primary component, and the slBF and cBF samples separated by the secondary component (see Additional file 2: Figure S7). Thus, stage-specific expression changes dominated any strain-specific differences between the two sets of BF samples.

Gene-level ribosome footprint read counts were compared across biological conditions to assess changes in the translational landscape across stages (Additional file 4 provides comparison data for all genes). As shown in Figure 3A, after excluding the 743 annotated pseudogenes, 1478 of the remaining 8398 genes had at least 2 -fold more ribosome footprint reads in slBF than in PCF and 1493 had at least 2-fold more in PCF than in slBF (using a false discovery rate (FDR) <0.01). In contrast, only 932 and 657 showed a statistically significant $>2$-fold increase in mRNA read counts for slBF and PCF respectively (Figure $3 \mathrm{~B}$ ). Thus, $\sim 35 \%$ of all genes showed statistically supported stage-regulated expression of protein production between these two conditions, while only $~ 19 \%$ showed similar changes in mRNA abundance. Of the former, $81 \%$ showed similar differences (i.e. $>1.5$-fold change in the same direction) in the $\mathrm{CBF}$ to PCF comparison, providing a high level of confidence that changes reflect stage-specific changes. As will be discussed later, the vast majority of genes that were up-regulated in slBF as compared to cBF encoded VSGs or expression site-associated genes (ESAGs, also often associated with antigenic variation in $\mathrm{BF}$ ), while those that were downregulated in slBF alone fell into several different functional categories.

Of the mostly highly translated genes (those ranked in the top $5^{\text {th }}$ percentile for ribosome footprint RPK in each stage, 701 in total) in slBF or PCF, 30\% are shared between both stages (as indicated by purple dots in Figure 3C). These include $\alpha$ - and $\beta$ - tubulins, translation elongation factor $1 \alpha$, aldolase, and glycerol 3-phosphate dehydrogenase. Of the remaining genes (indicated by magenta for slBF and green for PCF), 19\% show more than a 10 -fold difference in expression levels between the two stages. As expected, these include VSGs and procyclin (the major surface proteins of BF and PCF, respectively). Additionally, 23 of the 56 genes in the top $5 \%$ for protein production in slBF, but in the bottom $50 \%$ in PCF, encode proteins of unknown function. Similarly, 3 of the 9 genes that are in the top $5 \%$ in PCF, but the bottom $50 \%$ in slBF, encode hypothetical proteins of unknown function. Thus, these data highlight a set of unstudied genes that may play roles in parasite development. When the most highly expressed genes were separated into functional categories (see Methods), several categories showed differential protein production between slBF and PCF (Figure 3D). Proteins involved in translation were over-represented in among those up-regulated in PCF (including the ribosomal proteins seen in Figure 2D), while those involved in protein transport/modification and degradation were over-represented in those up-regulated in slBF. Proteins associated with DNA (mostly histones), metabolism (see below), organelle biogenesis (tubulins and flagellar proteins), and protein folding (HSPs and T-complex) were over-represented in the genes highly expressed in both stages as compared to their representation in the genome.

To visualize all substantial changes in mRNA abundance and translation between stages, we performed clustering analysis (based on fold-change in mRNA and ribosome footprint read counts) for all genes with at least a four-fold change in ribosome footprint reads between any two of the biological conditions. As shown in Figure 4, these genes segregated into four distinct clusters, each containing 2-3 sub-clusters. Cluster A contains 608 genes that are up-regulated in PCF, while clusters B, C and D contain 627, 185, and 135 genes, respectively, that are up-regulated both slBF and $\mathrm{cBF}$ (B), slBF alone (C) or cBF alone (D). Cluster A shows an over-representation of genes involved in metabolism and transport (see Figures 4 and Additional file 2: Figure S10), reflecting the up-regulation of oxidative phosphorylation and amino acid metabolism of PCF compared to BF $[47,48]$. In addition, sub-cluster A3 is enriched for the structural components of the cytoplasmic ribosome mentioned above, highlighting the variation in translational activity between PCF and SIBF, with intermediate levels in cBF. As expected, clusters B, C and D contain a large number of ESAGs that were up-regulated in both BF conditions (Figures 4 and Additional file 2: Figure S8). Numerous VSG genes are present in clusters $C$ and $\mathrm{D}$, reflecting both antigenic variation and extensive polymorphisms between the strains used. Interestingly, a disproportionate number of transporters, interacting proteins and proteases are up-regulated in either slBF, cBF or both (see Additional file 2: Figure S8). In addition, many genes involved in glycolysis, glycerol and lipid metabolism were up-regulated in BF (see Figure 4), although these categories are not significantly 


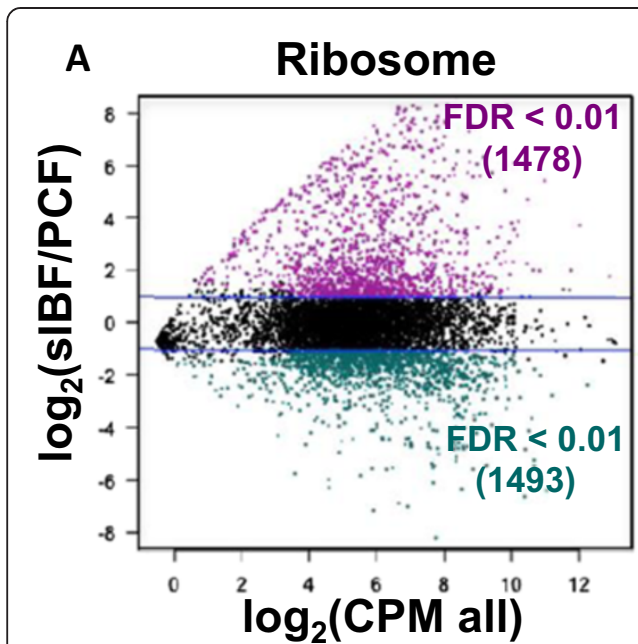

C

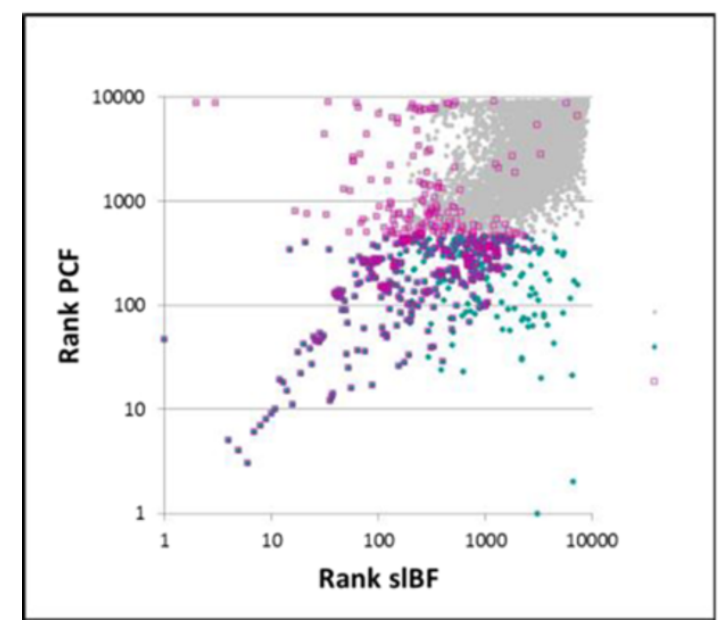

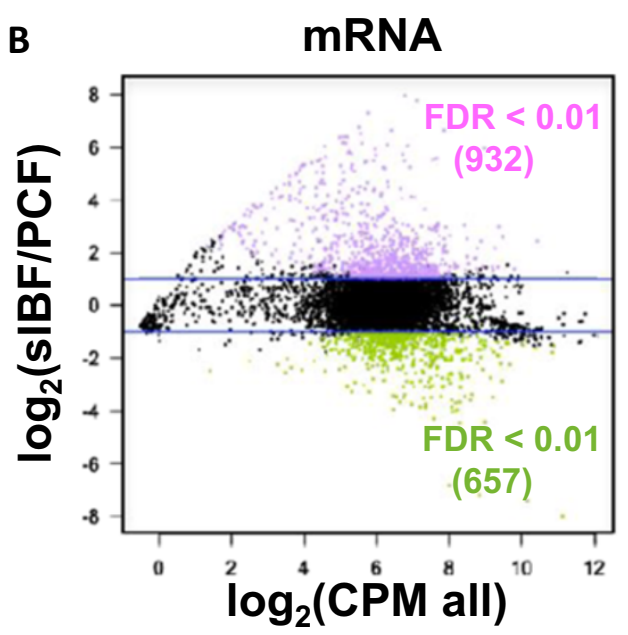

D

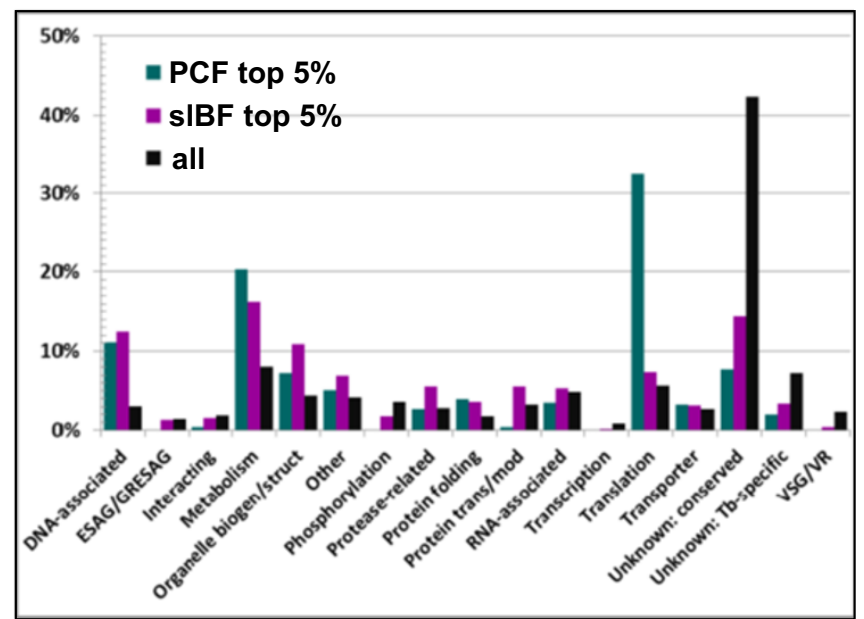

Figure 3 Ribosome profiling reveals extensive differential protein production. In this smear plot the fold change in read counts for ribosome footprint (A) and mRNA (B) were plotted against average read counts per million reads of the pooled libraries for sIBF and PCF. Dots that lie outside the blue lines are up-regulated at least 2-fold. Those that are statistically supported (FDR $\leq 0.01$ ) are colored (green/dark green for PCF and pink/magenta for sIBF). Note that almost twice as many genes (2971) up-regulated for protein production as compared to mRNA expression (1589). C) Stage-regulation of genes most highly expressed at the level of protein production. This dot plot depicts the gene rank for protein production in sIBF and PF. The rank is based on median ribosome footprint RPK in the biological replicates. Those in the top 5\% for sIBF are outlined in magenta, those in the top 5\% for PCF are green, and those that are in the top 5\% for both appear purple. The remaining genes are marked in gray. D) Categorization of most highly expressed genes compared to genome-wide representation. Top 5\% of slBF, magenta; top 5\% PCF, green; genome, black.

over-represented in clusters $\mathrm{B}, \mathrm{C}$ or $\mathrm{D}$ relative to the entire genome.

\section{Changes in translational efficiency}

Increased protein synthesis can be mediated by a change in mRNA level or translation efficiency, or a combination of both. Changes in mRNA levels are well known to be important during T. brucei development, with studies using different technologies and statistical cutoffs yielding estimates of 5-6\% ([6,9], 25\% [7] and 40\% [11] of genes as being differentially expressed between
cBF and PCF. However, changes in protein production (as measured by ribosome profiling) are generally higher than those in mRNA abundance, providing evidence for changes in TE being involved in regulation of differential gene expression. This is the case in terms of both the number of genes that were significantly differentially expressed between PCF and slBF (2971 ribosome footprint vs 1589 mRNA, see Figure 3A and B) and the magnitude of the change for individual genes (see Figure 4). Indeed, the sub-clusters in Figure 4 begin to segregate genes with similar changes in translation (ribosome 


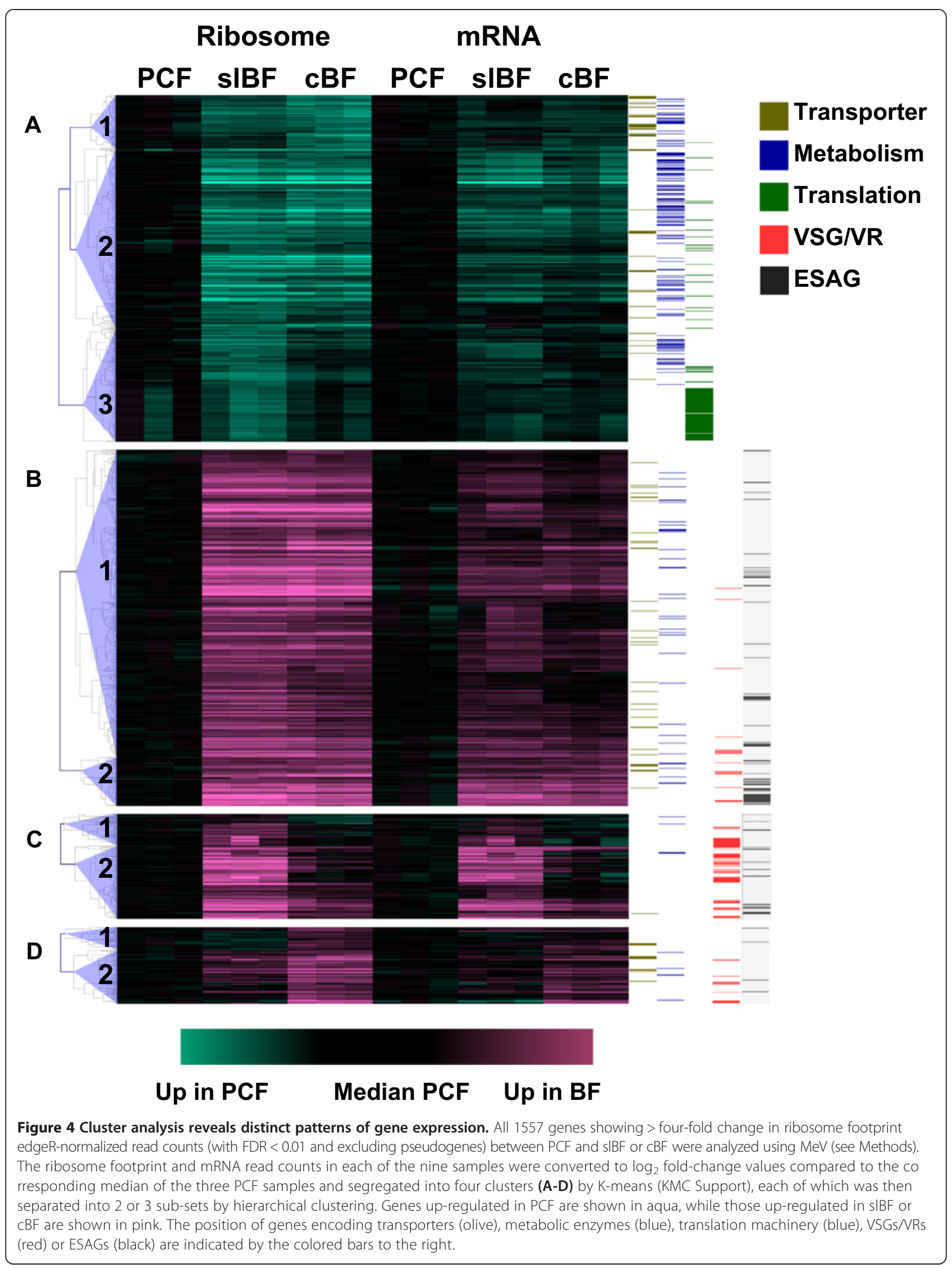


footprint) and mRNA (sub-clusters A2, B2, C2, and D2), from those where the change in translation was much greater than that in the mRNA level (sub-clusters A1, A3, B1, C1 and D1), although the separation is not complete. This variation in the contribution of changes in mRNA abundance and changes in TE to yield differences in protein production can be more readily seen by plotting these parameters for all genes (Figure 5A). In this representation, the grey dots (around the downward diagonal) represent genes for which there was no significant change in protein production (even though in some cases the mRNA level or TE may change), while the colored dots correspond to genes with at least a 2-fold change. The light-colored dots near the $\mathrm{x}$-axis indicate genes where changes in mRNA levels accounted for most (or all) of the change in translation, while the darkcolored dots near the $y$-axis represent genes where most of the change in translation was mediated by TE. There are also a large number of genes (indicated by the medium-colored dots near the upward diagonal) where both mechanisms appeared to play an important role.

We have identified a number of clear examples for each of these three categories of regulation. Two cases that illustrate regulation (primarily) by mRNA are shown in Figure 5B. The mRNA from Tb927.4.4740, which encodes a ceramide synthase-related protein, increased 3.6-fold in slBF (compared to PCF), accounting for most of the 4-fold increase in protein production. Similarly, the mRNA from Tb927.4990, which encodes the $\delta$ subunit of ATP synthase, was 4.4-fold higher in PCF, with a corresponding 5-fold increase in ribosome footprint read counts. Since most of the increase in translation was due to the change in mRNA abundance, the extent of protein production specified by these genes can be readily assessed by RNA-seq alone. However, for the other genes, RNA-seq provides only a partial (or even misleading) picture. Figure $5 \mathrm{C}$ shows two examples of regulation mediated primarily by change in $\mathrm{TE}$. Tb927.9.12740 (which encodes a protein with similarity to 2-phosphoglycerate kinase) had $\sim 16$-fold higher ribosome footprint read counts in slBF, while the mRNA read count increased by only $\sim 1$.7-fold. Similarly, the mRNA read count from Tb927.11.1340 (which encodes a protein with an atypical protein kinase domain) was slightly (1.3-fold) lower in PCF, but the ribosome footprint read count increased by $\sim 13$-fold. Thus, in both cases the change in TE (calculated to be $\sim 9$-fold and $\sim 16$-fold, respectively) accounted for most (or all) of the increased translation. Finally, the two cases in Figure 5D provide examples of where a combination of changes in both mRNA abundance and TE appear to play a role in regulating gene expression. Tb927.10.4770 (which encodes phosphatidyl inositol 4,5 kinase) showed a 5-fold increase in ribosome footprint reads in slBF, resulting from a 2-fold increase in mRNA reads and 2.4-fold increase in TE; while Tb927.10.15410 (glycosomal malate dehydrogenase) had 111-fold more ribosome footprint reads in PCF, resulting from a $\sim 12$-fold increase in mRNA and $\sim 6$-fold increase in TE.

In order to systematically and statistically assess the role of mRNA and TE changes in regulating gene expression, we applied a generalized linear model (GLM) framework within DESeq [49] to the raw CDS read count data from both mRNA and ribosome footprint. The GLMs corresponded to the potential regulatory mechanisms based on: a) mRNA abundance change only; b) TE change only; c) both mRNA abundance and TE change and d) no significant regulation. DESeq tested each gene individually for the fit to the models, and those that showed significant regulation were then assigned to model a, b, or c, as described in Methods. The GLM results, alone and in combination with edgeR criteria (2-fold difference in ribosome footprint reads, FDR $<0.01$ ), are shown in Table 1 . The results of these analyses for slBF versus PCF indicate that TE (either alone or together with changes in mRNA level) plays a substantial role in regulating stage-specific gene expression, accounting for $805 / 1290$ (62\%) of the genes that were more highly expressed in PCF and 996/1359 (73\%) of those more highly expressed in slBF. However, only a modest number of genes (59 in PCF and 126 in slBF) are predicted to be regulated by $\mathrm{TE}$ alone, although these analyses are likely complicated by the complex relationship of translation to mRNA decay [50], which may over-emphasize the role of changes in mRNA abundance. Importantly, when the same analyses were applied to cBF versus PCF, we obtained similar results (Table 1), except that (as indicated above) there were fewer stage-specific changes in gene expression in this situation, especially in terms of genes that were expressed at higher levels in PCF. Nevertheless, 769 genes were identified where GLM analysis showed that regulation of TE plays a significant role in stage-specific gene expression for both slBF and $\mathrm{cBF}$ as compared to PCF and for which edgeR analysis also showed significant stagespecific changes in ribosome footprint ( $\geq 2$ fold, FDR $<$ 0.01 ). These included at least 33 cases where there was no significant change in mRNA level (Table 2).

To determine whether different classes of genes were regulated by TE versus mRNA during parasite development, we grouped them into the broad functional categories described above (see Additional file 1: Table S4 and Additional file 2: Figure S9). As mentioned above, cytoplasmic ribosomal proteins (a subset of the "Translation" category) were over-represented in those genes more highly expressed in PCF, and TE appeared to play a prominent role in their regulation, although some genes in the Translation category were also regulated by mRNA 


\section{A}

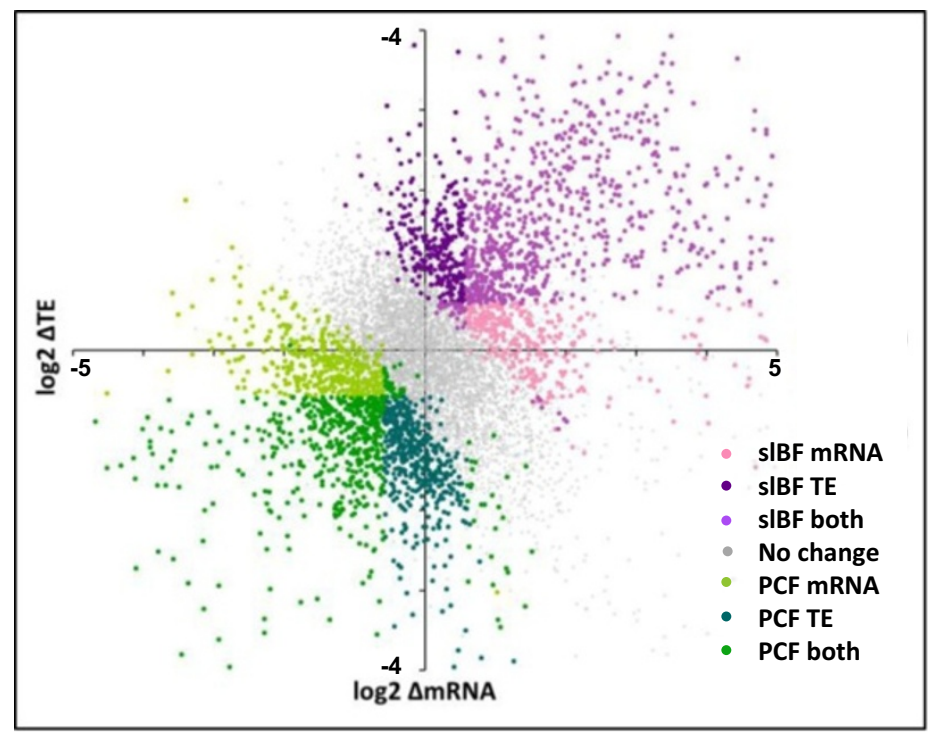

B

Tb927.4.4740
Ceramide synthas
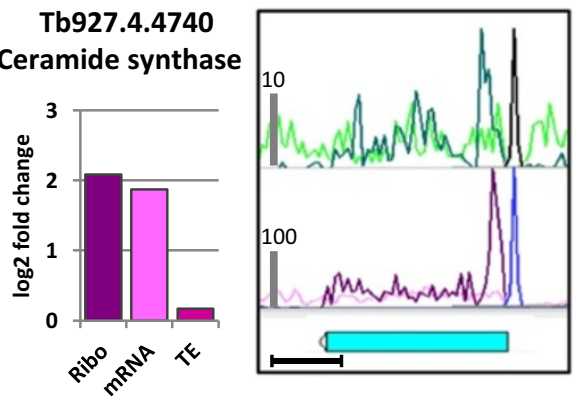

C

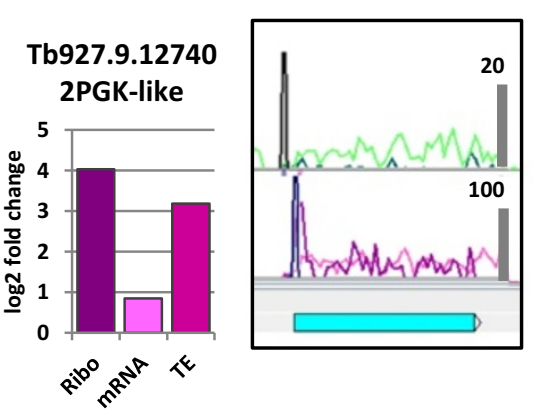

D Tb927.10.4770
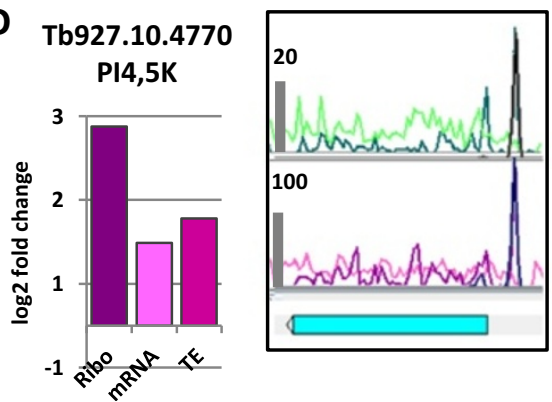
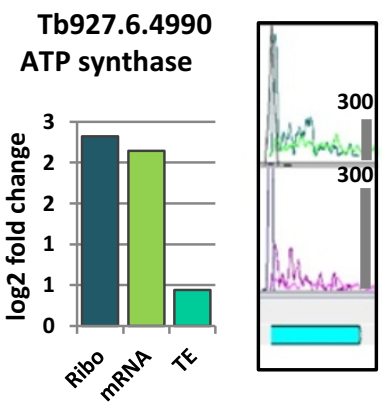

Figure 5 (See legend on next page.) 
(See figure on previous page.)

Figure $\mathbf{5}$ Regulation of gene expression at the level of TE and mRNA abundance. A) Genome-wide plot of the change in TE versus the change in mRNA between sIBF and PCF, expressed as log2 ratios. B-D) Examples of genes where changes in protein production are mediated by different mechanisms. Panel B, regulation primarily by changes in mRNA abundance; Panel $\mathbf{C}$, regulation primarily by changes in TE, and Panel D, regulation in which changes in both mRNA and TE contribute strongly. The histograms show the median log2-normalized fold change in read counts for ribosome footprint, mRNA and the TE. In the histograms, magenta tones are used for genes up-regulated in sIBF while green tones are used for those up-regulated in PCF. The Artemis view from PCF3 (green) and sIBF3 (magenta) are shown for each gene, with ribosome footprint being the dark color and mRNA being the light color. Similar changes were seen for cBF versus PCF. The genes depicted in this figure had negligible multi-mapping reads. The bars at the edge of the graphs indicate the relative scaling of ribosome profiling and mRNA read counts in the two stages. The SL reads (black in PCF, blue in sIBF) are not to scale. The scale bar below the first panel represents 500 nt, used for all images).

abundance. In contrast, genes encoding metabolic enzymes and proteins involved in protein folding that were up-regulated in PCF appeared to primarily use mRNA abundance to regulate their expression. Among the genes up-regulated in slBF, mRNA level appeared more important for VSG and ESAGs, while transporters were regulated more by change in TE. However, almost half of the genes that were regulated by $\mathrm{TE}$ alone encoded proteins with unknown (but conserved) function. We also observed that genes with the highest levels of protein production were more likely to exhibit stage-regulation (Additional file 2: Figure S10).

\section{uORFs and translational regulation}

In other eukaryotes, one of the mechanisms by which gene-specific changes in translation can be exerted is through the presence of uORFs that interfere with the translation of the main CDS [27,51-53]. Not all uORFs modulate translation, but evidence indicates that those that do act through being themselves translated (only

Table 1 Mechanisms of gene regulation

\begin{tabular}{lcccc}
\hline slBF:PCF & Stage $^{\mathbf{a}}$ & DESeq & Both DESeq and edge $^{\mathbf{b}}$ & \% \\
\hline no change & - & 5212 & 5749 & $68.5 \%$ \\
TE only & PCF & 62 & 59 & $0.7 \%$ \\
both & PCF & 872 & 746 & $8.9 \%$ \\
mRNA only & PCF & 654 & 485 & $5.8 \%$ \\
TE only & SIBF & 130 & 126 & $1.5 \%$ \\
both & SIBF & 1023 & 870 & $10.3 \%$ \\
mRNA only & SIBF & 445 & 363 & $4.3 \%$ \\
\hline cBF:PCF & Stage & DESeq & Both DESeq and edgeR & $\%$ \\
\hline no change & - & 6528 & 6671 & $79.5 \%$ \\
TE only & PCF & 16 & 15 & $0.2 \%$ \\
both & PCF & 607 & 538 & $6.4 \%$ \\
mRNA only & PCF & 212 & 185 & $2.2 \%$ \\
TE only & CBF & 124 & 124 & $1.5 \%$ \\
both & CBF & 737 & 714 & $8.5 \%$ \\
mRNA only & CBF & 174 & 151 & $1.8 \%$ \\
\hline
\end{tabular}

${ }^{a}$ stage with higher protein production.

${ }^{\mathrm{b}}$ concordance of edgeR analysis indicating 2-fold up-regulation for ribosome footprint read counts (FDR <0.01) and DESeq GLM model. Those that were not concordant were binned into the "no change" group in this column. some uORFs are efficiently translated) [27,51-53]. Initiation at a uORF can compete with initiation at downstream translation start sites, reducing translation of the main CDS. Alternatively, increased translation of a uORF that overlaps with the main CDS could also interfere directly with the initiation of other ribosomes at the main translation start site.

Analysis of the 5' UTRs for all genes using our 5' end mapping indicated that only 950 intact genes had potential uORFs, for a total of 3284 uORFs (see Additional file 5 for coordinates and read counts, and Figure 6A for example). The percentage of genes is smaller than that cited in a previous study [20] (11\% vs $22 \%$ ), likely due to the further refinement of $5^{\prime}$ UTRs that we performed (see Methods). Additionally, manual inspection of a subset indicated that some putative uORFs we identified cannot be confidently placed on the same transcript as the CDS, since there was an intervening SL site and the mRNA reads dropped to near-baseline just prior to this site (see Figure 6B for example), suggesting that this likely represents an over-estimate of the number of genes with genuine uORFs. A similar phenomenon has been observed in budding yeast when $5^{\prime}$ terminus sequencing and ribosome profiling data were combined to discover apparent uORFs that actually reflected distinct short transcripts [54].

Of the 2646 uORFs that did not overlap or abut the main CDS, RRSs could be calculated for only 458; the remainder have very low ribosome read counts on the uORF or read counts of zero for other values (see Methods and Additional file 5). A total of 322 genes had uORFs with an RRS $>2$, providing some indication of uORF translation (one example is shown in Figure 6A). Furthermore, as compared to mRNAs with no uORFs, mRNAs that contained putative uORFs with RRSs $>2$ showed $\sim 2$-fold lower TEs and mRNA read counts (with $\mathrm{p}<10^{-48}$ ) in all three biological conditions (see Table 3) and in mRNA RPK (not shown). The TE difference between the two groups persisted even when we accounted for difference in mRNA read count levels by analyzing only genes with mRNA read counts in the second and third quartiles (Table 3). Although we could not calculate the RRSs of uORFs that overlap the main CDS, these CDSs also showed a similarly low TE (not shown). 
Table 2 Genes with stage-regulated expression controlled primarily by $\mathrm{TE}^{\mathrm{a}}$

\begin{tabular}{|c|c|c|c|}
\hline GeneID & Product & Log2TE sIBF-PCF ${ }^{b}$ & Log2TE CBF-PCF \\
\hline Tb927.1.1580 & cytochrome c oxidase assembly factor SCO1/2 & -2.92 & -2.10 \\
\hline Tb927.1.1820 & PIN nuclease domain protein & 2.43 & 2.12 \\
\hline Tb927.1.3130 & protein kinase & 2.38 & 3.46 \\
\hline NTCDS.Tb3.NT.15 & hypothetical protein & 2.62 & 2.61 \\
\hline Tb927.4.2290 & glucose transporter & 1.95 & 2.22 \\
\hline Tb927.4.5190 & hypothetical protein, conserved & -3.11 & -3.54 \\
\hline Tb927.5.285b & receptor-type adenylate cyclase ESAG4 & -2.24 & -2.26 \\
\hline Tb927.5.320 & receptor-type adenylate cyclase GRESAG4 & -2.00 & -2.16 \\
\hline Tb927.5.430 & ISG65/75 domain protein & 2.47 & 1.69 \\
\hline Tb927.7.1840 & zinc finger protein & 2.70 & 1.97 \\
\hline Tb927.7.1880 & zinc finger protein & 2.82 & 1.99 \\
\hline Tb927.7.6150 & hypothetical protein, conserved & 3.65 & 3.52 \\
\hline Tb927.8.2480 & 3-oxo-5-alpha-steroid 4-dehydrogenase-like & 2.97 & 2.69 \\
\hline Tb927.8.2780 & RNA-binding protein RBP10 & 4.75 & 7.35 \\
\hline Tb927.8.4570 & RING domain protein & 2.39 & 1.88 \\
\hline Tb927.8.5480 & hypothetical protein, conserved & 2.10 & 2.19 \\
\hline Tb927.8.6130 & hypothetical protein, conserved & 2.63 & 1.95 \\
\hline Tb927.8.7500 & hypothetical protein, conserved & 2.18 & 2.45 \\
\hline Tb927.9.1500 & protein kinase & 1.58 & 3.52 \\
\hline Tb927.9.3100 & hypothetical protein, conserved & 2.09 & 2.57 \\
\hline Tb927.9.3820 & syntaxin & 1.88 & 2.03 \\
\hline Tb927.9.15850 & hypothetical protein & 3.67 & 3.30 \\
\hline NTCDS.Tb9.NT.51 & hypothetical protein & 2.53 & 2.49 \\
\hline Tb927.10.2210 & ubiquitin carboxyl-terminal hydrolase & -2.90 & -3.07 \\
\hline Tb927.10.12500 & P-type H -ATPase & -2.08 & -2.29 \\
\hline Tb927.10.14910 & sarcoplasmic reticulum sarcalumenin & 1.63 & 2.11 \\
\hline Tb927.11.1340 & Protein kinase-like domain protein & -3.96 & -2.71 \\
\hline Tb927.11.3630 & nucleobase/nucleoside transporter 8.1 & 4.28 & 2.16 \\
\hline Tb927.11.7820 & endonuclease/exonuclease/phosphatase & 1.94 & 3.12 \\
\hline Tb927.11.12730 & hypothetical protein & 3.00 & 2.73 \\
\hline Tb927.11.14740 & Recombinase-like domain protein & 1.70 & 2.08 \\
\hline Tb927.11.15840 & L-Lysine transport protein & 1.09 & 2.11 \\
\hline Tb927.11.15860 & L-Lysine transport protein & 1.14 & 2.05 \\
\hline
\end{tabular}

${ }^{\mathrm{a}} \mathrm{GLM}$ and edgeR both indicate TE plays a significant role and that mRNA change is not significant.

${ }^{\mathrm{b}}$ Positive values indicate higher TE in BF, negative indicate higher TE in PCF.

Thus, it is likely that uORFs reduce translation of a number of mRNAs in trypanosomatids, as in other organisms. Analysis of the functional category of uORFcontaining mRNAs revealed nothing remarkable, except for $\sim 2$-fold over-representation of $T$. brucei-specific genes and slight $(<2$-fold) under-representation of those involved in translation, transport, organelle biogenesis/ structure, and proteolysis, as well as VSGs.

We saw no enrichment for genes with uORFs among those that were classified by GLM as stage-regulated by TE (alone or in concert with regulation of mRNA abundance) (Additional file 2: Figure S11). Of the 13 genes that were regulated primarily by TE and had putative $\mathrm{uORFs}$, five showed somewhat higher TE in the stage in which the UORF had more ribosome footprint reads, while five had very few uORF reads in both stages, two lacked a true uORF, and one was extensively multimapping precluding further analysis. Thus, we were unable to find convincing evidence that the putative uORFs contribute to stage-regulation of translation between PCF and slBF. This data does not rule out the possibility that some uORFs confer a component of 
A

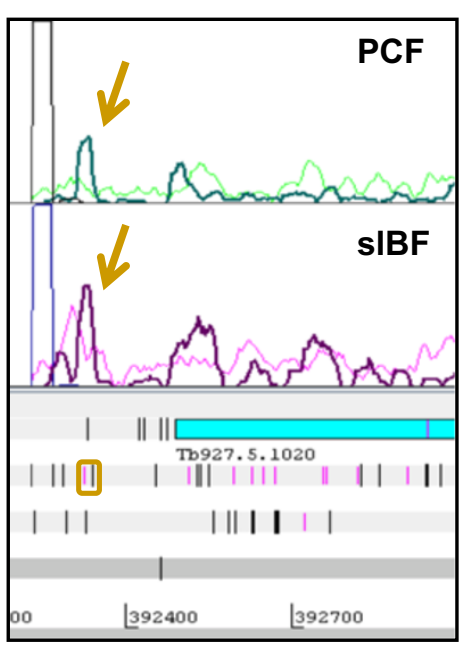

Tb927.5.1020
B

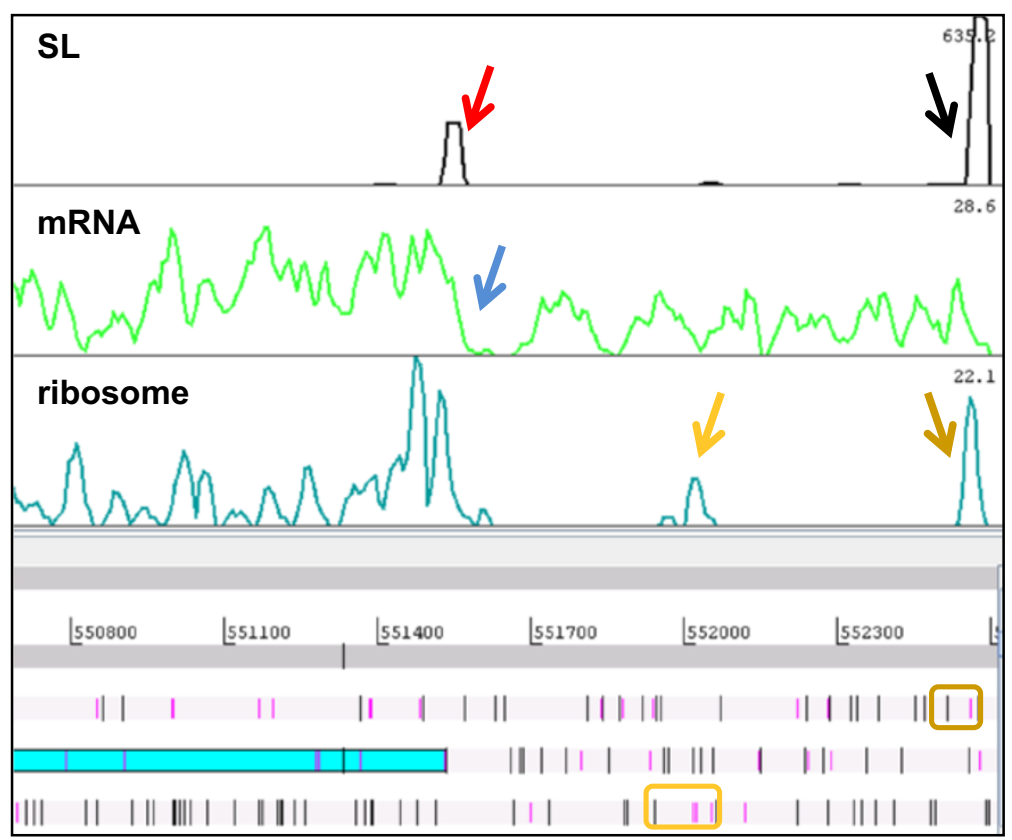

Tb927.11.1850

Figure 6 Predicted uORFs and translation. A) Example of a translated uORF that convincingly lies on the same transcript as the main CDS. A 5 aa UORF outlined in gold and delineated by the start codon (pink line) and a subsequent stop codon (black line) in the reading frame 2 is associated with the main CDS of Tb927.5.1020. It is translated in both stages (RRS =23.5). We do not see a significant difference in TE of the main CDS between stages (sIBF:PCF $\triangle \log 2 \mathrm{TE}=-0.25$ ). B) Candidate uORFs may not not lie on the same transcript as the CDS of Tb927.11.1850. Predicted UORFs are seen in all three reading frames downstream of the computationally predicted $5^{\prime}$ end of the mRNA defined by a peak in SL reads as described in Methods (black arrow). Two of these UORFs bear ribosome footprints with RRS scores $>70$ (gold arrows; ORFs are outlined on the map). The blue arrow marks a dip in the mRNA levels, followed by a second trans-splicing site just before the main CDS (red arrow). Thus, it is not convincing that most transcripts bearing the Tb927.11.1850 CDS also bear these putative uORFs. Data are shown for PCF3, although similar profiles were seen with sIBF.

stage-regulation, which could be revealed with further refinement of the transcriptome and translatome or by examination of other developmental stages.

\section{Conclusions}

The sequencing of the T. brucei genome in 2005 [55] ushered in an era of high-throughput analyses of the transcriptome [5-7,9-11] and proteome [2,3] of both insect and mammalian stages of this parasite. While these studies have been very informative and are transforming trypanosomatid research, both approaches have shortcomings that limit their usefulness for researchers in the field. Microarray and subsequent RNA-seq analyses have elucidated numerous changes in mRNA levels between life cycle stages at a comprehensive genome-wide scale, but they cannot identify genes that are regulated at the level of translational control. Conversely, mass spectrometry-based proteomic analyses suffer from lack of coverage, interrogating less than half of all cellular proteins. The recently developed technique of ribosome profiling [36,37] covers the middle ground by quantitatively interrogating mRNAs for the presence of ribosomes, thereby revealing the rate of translation for every gene. This ribosome-centric approach provides more specific quantitation and greater dynamic range than the mRNA-centric technique polysome profiling (coupled with microarray or other genomewide analysis), although it does not reveal distinct pools of mRNA that can be observed using latter approach.

Comparison of our results with those obtained from the most comprehensive published proteomic analysis comparing BF and PCF $[2,3,56]$, shows a good correspondence between changes in translation and protein level (see Additional file 2: Figure S12). Most (84\%) of the proteins that showed $>2$-fold up-regulation in $\mathrm{BF}$ had at least 1.5-fold up-regulation in protein production in one or both BF conditions used in this study. There was slightly less agreement (63\%) for proteins up-regulated in PCF, perhaps reflecting differences between strains and growth conditions used in the two studies. Some discrepancies are to be expected since the proteome is also modulated by individual protein stabilities, which would not be reflected in our data. While it is known that most abundant proteins are quite stable in PCF trypanosomes 
Table 3 The presence of uORFs and TE

\begin{tabular}{|c|c|c|c|c|c|c|}
\hline \multirow[b]{2}{*}{ All $^{\mathrm{a}}$} & \multicolumn{2}{|c|}{ PCF } & \multicolumn{2}{|c|}{ sIBF } & \multicolumn{2}{|c|}{$\mathrm{CBF}$} \\
\hline & $u O R F^{b}$ & no uORF & UORF & no uORF & UORF & no uORF \\
\hline Observations & 322 & 7009 & 322 & 7009 & 322 & 7009 \\
\hline \multicolumn{7}{|l|}{$\mathrm{TE}$} \\
\hline Mean & 0.3756 & 0.8434 & 0.3092 & 0.8031 & 0.3647 & 0.8678 \\
\hline$p^{c}$ & \multicolumn{2}{|c|}{$1.9 e-49$} & \multicolumn{2}{|c|}{$7.5 e-65$} & \multicolumn{2}{|c|}{$2.7 e-54$} \\
\hline \multicolumn{7}{|l|}{ mRNA } \\
\hline Mean & 522.4 & 1013.5 & 599.4 & 878.3 & 555.8 & 891 \\
\hline$P$ & \multicolumn{2}{|c|}{$1.9 e-07$} & \multicolumn{2}{|c|}{$1.2 \mathrm{e}-07$} & \multicolumn{2}{|c|}{$7.6 \mathrm{e}-06$} \\
\hline mRNA $(q 2+q 3)^{d}$ & UORF & no uORF & UORF & no uORF & UORF & no $u O R F$ \\
\hline Observations & 186 & 3476 & 161 & 3529 & 181 & 3512 \\
\hline \multicolumn{7}{|l|}{ TE } \\
\hline Mean & 0.3544 & 0.7239 & 0.3007 & 0.7648 & 0.3308 & 0.6912 \\
\hline$P$ & \multicolumn{2}{|c|}{$1.7 e-28$} & \multicolumn{2}{|c|}{$7.9 e-36$} & \multicolumn{2}{|c|}{$1.4 \mathrm{e}-29$} \\
\hline \multicolumn{7}{|l|}{ mRNA } \\
\hline Mean & 552.8 & 558.2 & 568.4 & 571.9 & 584.7 & 570.5 \\
\hline$P$ & \multicolumn{2}{|c|}{0.6514} & \multicolumn{2}{|c|}{0.8048} & \multicolumn{2}{|c|}{0.1144} \\
\hline
\end{tabular}

${ }^{a}$ Excluding pseudogenes and genes with unknown 5' UTRs.

${ }^{\mathrm{b}}$ Those genes with uORF RRSs $>2$.

'Mann Whitney U calculation.

${ }^{d}$ The genes were further restricted to those with mRNA levels in quartiles 2 and 3 for the biological condition analyzed (PCF 323-857, sIBF 334-902; cBF 350-85 median mRNA read counts).

[57], it is also likely that a subset of proteins are less stable, such as those required at specific points in the cell cycle. It is also interesting to note that changes in protein production had a greater magnitude than those in protein abundance, perhaps reflecting a larger dynamic range and sensitivity for ribosome profiling. This enabled us to detect 529 genes with a 10 -fold or greater change in protein production between stages, of which only 143 were detected by the proteomics approach.

While this manuscript was in preparation, another paper describing the application of ribosome profiling to T. brucei was published [20], including a comparison of single samples of $\mathrm{CBF}$ and PCF. That study revealed extensive changes in translation during parasite development, but lacked the biological replicates to provide a robust statistical analysis of the stage-regulated changes. Here, we compared three biological replicates of PCF from one strain (927) to three replicates of both in vivo derived slBF from the same strain and $\mathrm{cBF}$ of another strain (427). When contrasting the two studies, $R^{2}$ values of $\sim 0.63$ were seen when comparing PCF and cBF ribosome footprint data, with stronger correlations for mRNA data $\left(\mathrm{R}^{2}\right.$ of 0.68 and 0.85 for PCF and $\mathrm{cBF}$ respectively) (Additional file 2: Figure S13A). Of the 27 genes specifically noted by Vasquez et al. [20] as showing the highest level of translational regulation, 24 showed at least a 2-fold change in TE in our comparison of slBF vs PCF. Conversely, over $65 \%$ of genes with at least a 4-fold increase in $\mathrm{TE}$ in $\mathrm{BF}$ in our experiments also showed at least a 2-fold change in the published study (Additional file 2: Figure S13B). Given the likely differences in growth conditions, strains, and data analysis these similarities strengthen the conclusions that translational regulation is important in the development of these parasites. Moreover, our use of biological replicates revealed several phenomena that were not previously apparent.

Firstly, we observed that while the mRNAs for genes encoding structural components of the cytoplasmic ribosome were relatively abundant under all conditions, their translation efficiency varied considerably between samples, both within and between conditions, and they were relatively poorly translated. Low rates of translation for mRNAs encoding ribosome-associated proteins is a well-known phenomenon in other organisms, including under conditions of cell stress $[58,59]$. This may explain the somewhat surprising observation that the TE of ribosomal proteins was lower in slBF than PCF, since although slBF grow more rapidly than the cultured insect form, they are exposed to stresses in vivo. Additionally, the commitment of some slBF parasites to exit the cell cycle to become stumpy forms (even though the populations were $>95 \%$ morphologically slender) may contribute to the reduced translation of ribosome proteins. This argument is buttressed by the finding of an intermediate 
TE for ribosomal proteins in cBF derived from a monomorphic strain (T. brucei 427), which does not differentiate into stumpy forms in vitro or in vivo, but are nonetheless highly sensitive to cell density. Thus reduced translation of ribosomal proteins in T. brucei may be an early event when proliferation slows. In many mammalian cells and in maize, regulation of ribosomal protein production appears to rely on pyrimidine rich elements at the 5 ' end of the mRNAs known as TOP elements [59-61]. However, in $T$. brucei polypyrimidine tracts are signals for trans-splicing (and are removed during processing) and mRNAs are all identical at their $5^{\prime}$ termini. We examined the $5^{\prime}$ UTRs of the cytoplasmic ribosomal proteins and saw no enrichment of pyrimidines (43\% CT as compared $45 \%$ for all transcripts, exclusive of the common $5^{\prime} \mathrm{SL}$ sequence, see Additional file 2: Figure S14). Thus, the mechanism of translational regulation of these structural proteins of the ribosome must differ in trypanosomes. However, we detected no enriched motifs in the $5^{\prime}$ UTRs as compared to the overall transcriptome (by MEME analysis, [62]), although the ribosomal protein 5' UTRs are shorter than average (median of $21 \mathrm{nt}$ as compared to 87 nt median for all genes). As regulation of ribosome biogenesis is a central part of stationary phase development in many organisms and if the trypanosome must modulate the TE of ribosomal proteins during its life cycle, then the mechanisms underlying this regulation, which appear to differ from the analogous control of animal ribosomal protein translation, may present a therapeutic target. Future studies dissecting the mechanism of this control in trypanosomes will therefore prove interesting.

Our results also revealed that regulation of protein production in $T$. brucei is more extensive than previously anticipated from changes in mRNA abundance. While just under two hundred genes appeared to be regulated by changes in TE alone, several thousand show changes in translation substantially larger than the changes in their mRNA level. It is possible that some of these changes in TE reflect alternative splicing, which can be further investigated using existing and our updated SL data, although additional experimentation will be required. It will also be interesting to compare ribosome profiling of slBF with stumpy BF, which are growtharrested forms that are poised for transformation into PCF upon ingestion by the tsetse fly. Previous microarray analyses [6,7], demonstrated that few mRNAs differ in abundance between stumpy BF and slBF, but early studies indicated that translation is much reduced in stumpy BF [63] and microarray analysis of polysome fractions has identified a subset mRNAs that are differentially translated between the two stages [19]. Ribosome profiling has greater sensitivity in revealing changes in protein production than does polysome analysis, so we might expect that under conditions of limited translation (such as stumpy BF), more genes will be revealed as translationally regulated. By analysis of different additional stages and conditions, it is likely that different groups of genes under translational control will be revealed, potentially operating through different mechanisms.

\section{Methods}

\section{Parasites and cell extracts}

The pleiomorphic T. brucei strain TREU927, which has the most complete genome sequence at present [55], was employed for production of slBF and PCF. Three biological replicates of each stage were used (see Additional file 1: Table S1). slBF were grown in irradiated Wistar rats following injection of $10^{8}$ parasites derived from stabilates following IACUC approved protocols. The parasites were harvested on day 3 at a parasitemia of $5 \times 10^{7}-1 \times 10^{8}$ cells per $\mathrm{ml}$. Only parasite populations with greater than $99 \%$ slender cells were used. After harvest, the blood was centrifuged and the buffy coat extracted and placed into $20 \mathrm{ml}$ HMI-9 medium (without serum) pre-warmed to $37^{\circ} \mathrm{C}$. To arrest translation, cycloheximide was added to $100 \mu \mathrm{g} / \mathrm{ml}$ and incubated for 2 minutes at $37^{\circ}$. To rapidly chill the cells, $300 \mathrm{ml}$ of ice-cold phosphate buffered saline with glucose (PSG) was added and the cells were pelleted at $4^{\circ}$. Parasites from 2-3 animals infected from the same culture of in vitro grown parasites were pooled and lysates prepared as described below. Microscopic analysis showed that rat white blood cells represented less than $1 \%$ of the population. For $\mathrm{cBF}$, a derivative of $T$. brucei monomorphic strain Lister 427 was grown in vitro in HMI-9 medium [64] and harvested when the cultures were between $8 \times 10^{5}$ and $1.6 \times 10^{6}$ parasites $/ \mathrm{ml}$. The cultures were centrifuged for 10 minutes at $900 \times \mathrm{g}$, resuspended in $25 \mathrm{ml}$ of pre-warmed serum-free medium, treated with cycloheximide and rapidly chilled as above. Three biological replicates of these in vitro derived $\mathrm{cBF}$ were used for comparative purposes. We grew strain 927 PCF in SDM79 medium containing glucose [65], with $2-4 \times 10^{9}$ parasites being harvested in mid-log phase (density of $5 \times 10^{6}-1.2 \times 10^{7}$ cells $/ \mathrm{ml}$ ). The initial large volume of culture was centrifuged at $5000 \times \mathrm{g}$ for 5 minutes at room temperature and the pellet resuspended in $50 \mathrm{ml}$ of medium lacking serum. The parasites were incubated for $2 \mathrm{~min}$ in cycloheximide as above, rapidly chilled by the addition of $250 \mathrm{ml}$ PSG, and collected by centrifugation.

Cell pellets were resuspended in Buffer A (10 mM Tris $\mathrm{pH} 7.4,300 \mathrm{mM} \mathrm{KCl}, 10 \mathrm{mM} \mathrm{MgCl}_{2}$, plus protease inhibitors [63]) to approximately $1.3 \times 10^{9}$ cells $/ \mathrm{ml}$. Approximately one-third of the sample was placed into TRIzol (Life Technologies) for RNA extraction following the manufacturer's suggested protocol. To the remainder, one-sixth volume of buffer A containing $0.2 \mathrm{M}$ sucrose and $1.2 \%$ Triton $\mathrm{N}-101$ was added and the 
samples were homogenized (30 strokes using a chilled dounce with a 0.004-0.006 inch clearance pestle). After transfer to a pre-chilled microfuge tube, the samples were clarified by centrifugation in a microfuge at $15,000 \mathrm{rpm}$ for one minute. The supernatant was withdrawn, pooled if needed, and then aliquots flash frozen in liquid nitrogen for storage at $-70^{\circ} \mathrm{C}$. These extracts were then used for ribosome footprinting or polysome gradients. Polysome analysis was performed as previously described [63].

\section{Library preparation and sequencing \\ Ribosome footprinting}

Preliminary experiments established the appropriate conditions for RNAse I treatment of lysates (Additional file 2: Figure S1A). After thawing on ice, RNase I (Ambion) was added at 30 units $/ \mathrm{OD}_{260}$ of lysate. Samples were then incubated for 1 hour at room temperature. RNase digestion was stopped by adding 400 units RNasin (Promega). Samples were them layered over a $1 \mathrm{ml} 1 \mathrm{M}$ sucrose cushion prepared in buffer $\mathrm{A}$ and ribosomes were pelleted by centrifugation for 4 hours at 70,000 $\times \mathrm{g}$ in an SW55 rotor. After removing the supernatant, the ribosomal pellet was resuspended in $500 \mu \mathrm{l}$ buffer $A$ with $10 \mathrm{mM}$ EDTA replacing the $\mathrm{MgCl}_{2}$ to dissociate the ribosomes (Additional file 2: Figure $\mathrm{S} 1 \mathrm{~B})$. The protected fragments were then separated from contaminating larger ribosomal RNA fragments by passage through an Amicon Ultra-4 or YM-100 column with 100,000 MW cut-off. The RNA in the flowthrough $(400 \mu \mathrm{l})$ was extracted with phenol: $\mathrm{CHCl}_{3}$ :isoamyl alcohol and the RNA precipitated.

\section{mRNA libraries}

Poly $(\mathrm{A})+$ RNA was isolated using Dynabeads mRNA Direct (Life Technologies). RNA was fragmented as described [36] and fragments between 30 and 70 nucleotides isolated. For a detailed protocol on generating sequencing libraries for both the ribosome protected and fragmented mRNA library see Ingolia et al. [66]. Briefly, following dephosphorylation the adapter Linker-1 (IDT) was ligated to the 3 ' end of the fragment and the ligated product gel purified. The adapter was used for priming reverse transcription with the primer $\mathrm{RP}_{-}$ index_RT (all primers are provided in Additional file 1: Table S6). Following gel purification the cDNA was circularized with Circ Ligase (Epicenter Biotechnologies). Circles containing ribosomal RNA were subtracted using biotinylated primers at $10 \mu \mathrm{M}$. The final library was generated by PCR using RP_index_PCR_forward and one of the RP_index reverse primers.

\section{SL RNA-seq libraries}

Libraries enriched for the $5^{\prime}$ ends of mRNAs were constructed from three biological samples of strain 927 (two PCF and one slBF of the biological samples above) and one cBF sample from strain 427 , as described previously [67]. In brief, RNA was prepared and cDNA synthesized using primer Random5. Second strand synthesis was primed using $\mathrm{SL} \_2^{\text {nd }}$ primer3, which matches the $3^{\prime} T$. brucei SL sequence. The sequencing library was generated by PCR using the primer Multi-PCR P2 and one of the RP_index_PCR_reverse primers.

All libraries were sequenced using Illumina GA II machines at the High Throughput Genomics Unit at the University of Washington to generate $\sim 36$ nt reads using the proprietary Illumina read 1 sequencing primer (Rd1 SP) for fragmented mRNA and ribosome profiling libraries, or a custom sequencing primer (SL_SEQ_Primer2) for the SL RNA-seq libraries, as well as the Illumina indexing sequencing primer (Index SP).

\section{Bioinformatics}

Reads were assessed for their average quality, average GC, base composition, and variability between clusters, and those with average quality less than 30 were removed. T. brucei strain 927 genome sequences and gene annotations (version 5.0) were downloaded from TriTrypDB. This version of $T$. brucei genome consists of 11 large chromosomes, plus a variety of other contigs (a number of short BAC contigs, 1 bin chromosome for Chr9, and two fork chromosomes for Chr11). We determined that $>95 \%$ of the genes present in these other contigs are repetitive genes and sub-telomeric genes that were already present on one of the main chromosomes, so only the 11 large chromosomes were considered for the read alignment, as the other contigs would only increase ambiguity in the alignment without providing any extra information.

The fast sequence files were aligned against the 11 chromosome sequences using Bowtie2 [68] in local mode (which allowed us to avoid trimming of adapter sequences from ends prior to alignment), using the

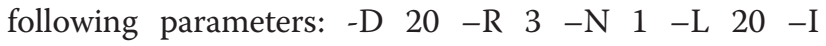
$\mathrm{S}, 1,0.50$. This allowed a maximum of one mismatch within a 20 nt seed region and a maximum of two mismatches across the entire alignment. The BAM files were sorted, indexed and reads mapping to structural RNAs were segregated in a separate BAM file to allow convenient viewing of the remaining reads in Artemis [69]. RNA sequencing data for ribosome footprint, mRNA, SL mapping has been deposited in the GEO database with the accession number GSE57336, with details of the biological samples deposited in the Bioproject database under accession number PRJNA246300. The RNA-seq data and revised annotations have been provided to TriTrypDB and Wellcome Trust Sanger Center respectively to be integrated into their genome databases.

The periodicity of ribosome footprinting was assessed as follows. For each codon (or in-frame triplet for those 
positions upstream of the start codon), the percentage of reads commencing at the first, second, or third position of that codon is plotted. Read counts for each codon/ triplet were the sum of all 28 and 29 nt reads for all CDSs in specific libraries. Similar calculations were performed for mRNA reads.

\section{Defining the $5^{\prime}$ end of transcripts}

Reads from SL RNA-seq libraries from PCF1 and slBF1 biological samples $(16.8 \mathrm{M}$ reads and $14.4 \mathrm{M}$ reads respectively) were aligned against the genome using Bowtie with following parameters (-e 70 -1 23 -n 2 -M 1 ). The major SL site (i.e., that with the most mapped reads upstream of the stop codon) for each CDS was identified, as well as other predominant SL sites located further $5^{\prime}$. The $5^{\prime}$ boundary of each mRNA was defined the most $5^{\prime}$ site with a read abundance of at least $40 \%$ of the major SL site, and a minimum of $20 \%$ of all SL sites for that CDS (from stop codon of upstream gene to the stop codon of the gene of interest). However, if there was an obvious gap in the mRNA read coverage, upstream SL sites were not considered and the next SL site was used. Of the 8398 intact genes, 96\% were assigned 5' UTRs and $84 \%$ of these we considered high confidence (defined of at least $50 \%$ of the SL reads for that gene, with at least 20 reads at that site). These included 904 high confidence 5 ' UTRs for genes lacking defined 5' UTRs in TriTrypDB and 758 high confidence changes versus those listed in TriTrypDB. A list of the mapped 5' UTRs used in this study is provided in Additional file 6.

\section{CDS refinement}

An iterative process used to refine the CDSs in the T. brucei strain 927 genome prior to the final assignments of read counts to individual genes. We manually inspected each chromosome for discrepancies from the annotated genome by visualizing SL, mRNA, and ribosome footprint reads from slBF and PCF libraries. New CDSs were added when ribosome footprint reads mapping throughout an ORF that commenced with a canonical start codon and was accompanied by an upstream SL and by mRNA reads (coordinates are provided in Additional file 3). Of these 120 corresponded to CDSs on recently discovered transcripts [10] and were given provisional GeneIDs commencing with "NTCDS". And additional 62 novel CDSs were also defined and were given provisional GeneIDs commencing with "NCDS". Boundaries for 573 CDSs were extended or shortened based on SL mapping of the start of the transcript by using the $5^{\prime}$-most in-frame start codon after the major SL site unless ribosome profiling or mRNA levels indicated otherwise. Many of these revised start codons had been previously noted
$[9,11]$, but had not yet been incorporated into the gene models in TriTrypDB v5.0. A comparison of these datasets is provided in Additional file 7. Briefly, 249 matched both of the other datasets and 237 matched one of the other datasets. A total of 249 CDSs were changed in only one dataset and merit further study. We deleted all CDSs annotated as "hypothetical protein, unlikely", as well as some annotated as conserved hypothetical proteins, that had no evidence of coding potential, according to one or more of the following criteria: 1) most mRNA reads mapped to the wrong strand, 2) the CDS mapped to the 3' UTR of another gene, showed similar mRNA read depth and lacked its own SL reads, 3) the CDS contained significant gaps in mRNA coverage, and/or 4) contained abundant internal SL reads (listed in Additional file 8). Genes that lacked sufficient information for discrimination were not eliminated; most of these lay within strand switch regions or close to the ends of chromosomes. These changes will be further discussed in another manuscript.

RRSs were calculated for all CDSs based on raw reads as described [38] and are provided in Additional file 3. The 3' UTR was defined as in TriTrypDB, or if data were lacking, as $100 \mathrm{nt}$ downstream of the stop codon, or to nt -13 relative to the next CDS, whichever was smaller. Briefly, for each CDS, the summed read counts across all ribosome profiling libraries and across all mRNA libraries were used to calculate as follows: (CDS ribosome footprints/3' UTR ribosome footprints)/(CDS mRNA/3' UTR mRNA). Only reads completely contained in the relevant region were included. For cases in which no ribosome footprints were seen in the 3' UTR, a value of 1 was substituted (to avoid divide-by-zero errors and yield a minimal RRS). For cases in which no mRNA reads were obtained for either the CDS or 3' UTR, an RRS cannot be computed.

\section{Normalization and read count assignment}

By analysis of the peak of ribosome footprint reads at the start codon, we determined that the footprint extended $12 \mathrm{nt}$ upstream of the first base of the codon being translated (Additional file 2: Figure S3), as previously observed in Saccharomyces cerevisiae [36]. Read counts for each CDS were generated using the HTSeq package [70] and included those from nt +46 ( 1 being the A of the ATG start codon) to the stop codon. For the 5' UTR, reads from the 5' end of the mRNA (excluding the SL) to nt -16 were included. Reads partially overlapping CDS or 5' UTR regions were included in the counts. The percentage of multi-mapping reads for each CDS is provided in Additional file 3. Raw read counts from each library type (mRNA, ribosome profiling) and each region (CDS region and UTR region) were tested for their differential expression in PCF, slBF and cBF samples using 
edgeR [40] and all nine biological samples (PCF, slBF, and $\mathrm{CBF}$ ) were normalized coordinately. To avoid overemphasizing fold-changes for genes with very low/zero read counts, 5 reads were added to the count for each CDS and 5' UTR region prior to normalization. edgeR was also used to correct for sample-specific variation, normalizing the data based on sequencing depth (total read counts per sample) and RNA composition (weighted trimmed mean of log expression ratios -TMM). These normalized read counts were used in our further TE calculations and unpaired t-tests were used to estimate variation within biological replicates. As a second means of estimating variation within biological replicates, the negative binomial model was fit under GLM framework onto the raw reads, along with corresponding scaling factors for each sample. From this fit, common, trended and gene-wise dispersions (biological coefficient of variation) were estimated using the corresponding functions in edgeR. The raw read counts along with sample specific scaling factors, gene-wise dispersions were fed into edgeR's GLM framework and a GLM likelihood ratio test was performed to identify differentially expressed genes.

\section{Cluster analysis of changes in gene expression}

All CDSs (excluding pseudogenes) that showed $>$ fourfold change in ribosome footprint edgeR-normalized read counts, with statistical support of FDR (Benjamini and Hochberg method) $<0.01$, in pairwise comparisons between PCF, slBF and/or cBF were analyzed using $\mathrm{MeV}$ [71]. The ribosome footprint and mRNA read counts for these 1559 CDSs from each of the nine samples were converted to $\log _{2}$ fold-change values compared to the corresponding median of the three PCF samples before being imported into $\mathrm{MeV}$. The genes (but not samples) were segregated into clusters using KMC Support with the following parameters: Distance Metric $=$ Pearson Correlation; Means or Medians = K-means; K-means repetitions $=50$ runs, with $80 \%$ threshold for occurrence in the same cluster; K-means run parameters $=4$ clusters, 50 iterations. Hierarchical Trees were also constructed using HCL based on Pearson Correlation using complete linkage clustering of genes only, with optimization of Gene Leaf Order. Different distance thresholds $(0.69-1.14)$ were then used to separate each of the four KMC Support clusters into 2 or 3 subclusters.

\section{TE calculation and identification of regulatory mechanism}

Median values of read counts for edgeR-normalized mRNA and ribosome footprint read counts were used to represent each gene in each stage. The TE (ratio of ribosome footprint to mRNA read counts) for each gene was calculated for each sample and the median TE of those values for each condition was used. Gene-level fold changes between biological conditions for TE, mRNA and ribosome footprint and their $\log _{2}$ ratios calculated.

We used DESeq [49] to apply GLMs to the raw count data from CDS regions (of both fragmented-mRNA and ribosome profiling experiments). The four generalized linear models used corresponding to the following potential regulatory mechanisms: a) mRNA abundance change only, b) TE change only, c) full model (both mRNA abundance and TE change), and d) no (significant) regulation. The raw count data from each gene was tested for its fit to each model and a deviation score was calculated given the average read counts. If the fit to the full model was better than the noregulation model (with statistical support of FDR $<$ 0.01 ), the gene was further analyzed. To be categorized as regulated by mRNA abundance, the following three criteria had to be met with statistical support: 1) the fit to the mRNA model was statistically significantly (FDR < 0.01 ) better than to the no-regulation model; 2) the TE model was not significantly better than the no-regulation model; and 3) the full model was not significantly better than mRNA model. The same logic was followed to assign genes to the TE model. The remaining genes were all assigned to the full model.

\section{Gene categories and descriptions}

Due to the divergence of trypanosomatids from humans and model organisms, many genes lack GO terms or are mis-categorized. Therefore, we manually updated our previous functional categorization of genes into molecular categories [7] and, when possible, subcellular categories. This was accomplished by reviewing the gene descriptions and user comments of all genes on TriTrypDB, as well as examination of the literature. In some cases, the presence of InterPro domains on "hypothetical" proteins allowed presumptive functional categorization.

\section{uORFs}

We developed an ad-hoc PERL script, which contains the get_orf algorithm from the EMBOSS package [72], to search for uORFs. No minimum size was specified. The region between the major SL addition site and the main CDS start codon was scanned for additional ATG start codons and, if one was encountered, the UORF was extended 3' to the next in-frame stop codon. For assignment of reads, the entire uORF was utilized. RRSs were calculated as above, except that reads were counted in the $\mathrm{UORF}$ if they were fully contained in the region from nt -12 (relative to the ATG) to $6 \mathrm{nt}$ past the stop codon. Reads were included in the 3' UTR counts if they were fully contained in the region from the stop codon until $13 \mathrm{nt}$ before the main CDS start codon or until the next ATG in any reading frame, whichever came first. 


\section{Additional files}

Additional file 1: Table S1. Biological samples. Table S2. Library statistics. Table S3. Median reads per kb across coding regions and UTRs from representative libraries. Table $\mathbf{S 4}$. Mechanism of stage-specific regulation of protein production, by gene category. Table S5. Primers.

Additional file 2: Figure S1. Experiments related to setup of system and 3 nt periodicity of ribosome footprints. Figure S2. A) Technical and biological replicates B) Distribution of read counts in representative mRNA and ribosome profiling libraries. Figure S3. Correlations between raw read count values of individual samples. Figure S4. Relative read counts around start and stop codons. Figure S5. Lack of ribosome profiling signal in 3' UTRs known to interact with RNA binding proteins. Figure S6. Polysome analysis for selected genes with differing TEs. Figure S7. High level clustering analysis of ribosome profiling samples. Figure S8. Enrichment of functional categories in different gene clusters.

Figure S9. Enrichment of functional categories regulated by different mechanisms. Figure S10. More frequent regulation of genes with high protein production. Figure S11. Genes with uORFs show similar regulation to all genes. Figure S12. Comparison of our data with Vasquez [20]. Figure S13. Correlation between changes in translation and protein abundance. Figure S14. Length and composition of 5' UTRs of ribosomal genes compared to rest of the genome.

Additional file 3: Coordinates, read count data, and RRSs for all CDSs. Additional file 4: Gene-level comparison of ribosome footprint, mRNA, and TE across biological samples.

Additional file 5: Genes with uORFs, uORF read counts, and uORF RRSs.

Additional file 6: CDS 5' UTR mapping.

Additional file 7: CDS start codons used in this study compared to TriTrypDB and other data.

Additional file 8: Genes removed from analyses with rationale and RRSs.

\section{Abbreviations}

BF: Bloodstream forms; CBF: Cultured bloodstream forms; CDS: Coding sequence; ESAG: Expression site-associated gene; FDR: False discovery rate; GLM: Generalized linear models; PCF: Procyclic cultured forms; RPK: Reads per kB; SL: Spliced leader; sIBF: Slender bloodstream forms; uORF: Upstream open reading frame; TE: Calculated translation efficiency; VR: Variant surface glycoprotein-related; VSG: Variant surface glycoprotein.

\section{Competing interests}

The authors declare that they have no competing interests.

\section{Authors' contributions}

BCJ prepared the biological samples and libraries for sequencing, and aided in experimental design and CDS revisions. GR devised data analysis tools and implemented them for the study, and aided in CDS revisions. EJRV assisted in tool development and bioinformatic analyses and CDS revisions. NTI provided critical advice on experimental design and data analysis. PJM and MP assisted in CDS revisions, conceived of the project, and supervised the experimental design and analyses with emphases on bioinformatics and trypanosome biology respectively. All authors contributed to writing and revision of the manuscript. All authors read and approved the final manuscript.

\section{Acknowledgements}

The authors thank Andrew Haydock for excellent technical assistance in the early phase of this work. NTI is supported in part by the Searle Scholars Program. EJRV is supported by a fellowship from the Conselho Nacional de Desenvolvimento Científico e Tecnológico (CNPq), Brazil (process ID: PDE 202223/2012-4). This work was funded in part by a grant from the National Institutes of Health, 1R21 Al094129. The authors are solely responsible for the content of this manuscript.

\section{Author details}

'Seattle Biomedical Research Institute, 307 Westlake Ave N, Seattle, WA 98109-5219, USA. ²Department of Molecular and Cell Biology, University of
California, Berkeley, CA 94720-3202, USA. ²Department of Global Health, University of Washington, Harris Hydraulics Building, 1705 NE Pacific St \#310E, Box 357965, Seattle, WA 98195, USA. ${ }^{4}$ Department of Biomedical Informatics and Medical Education, University of Washington, Seattle, WA 98195, USA.

Received: 21 September 2014 Accepted: 9 October 2014

Published: 20 October 2014

\section{References}

1. Kramer S: Developmental regulation of gene expression in the absence of transcriptional control: The case of kinetoplastids. Mol Biochem Parasitol 2011, 181:61-72.

2. Urbaniak MD, Guther ML, Ferguson MA: Comparative SILAC proteomic analysis of Trypanosoma brucei bloodstream and procyclic lifecycle stages. PLoS One 2012, 7:e36619.

3. Urbaniak MD, Martin D, Ferguson MA: Global quantitative SILAC phosphoproteomics reveals differential phosphorylation is widespread between the procyclic and bloodstream form lifecycle stages of Trypanosoma brucei. J Proteome Res 2013, 12:2233-2244.

4. Diehl S, Diehl F, El Sayed NM, Clayton C, Hoheisel JD: Analysis of stage-specific gene expression in the bloodstream and the procyclic form of Trypanosoma brucei using a genomic DNA-microarray. Mol Biochem Parasitol 2002, 123:115-123.

5. Koumandou VL, Natesan SK, Sergeenko T, Field MC: The trypanosome transcriptome is remodelled during differentiation but displays limited responsiveness within life stages. BMC Genomics 2008, 9:298.

6. Kabani S, Fenn K, Ross A, Ivens A, Smith TK, Ghazal P, Matthews K: Genome-wide expression profiling of in vivo-derived bloodstream parasite stages and dynamic analysis of mRNA alterations during synchronous differentiation in Trypanosoma brucei. BMC Genomics 2009, 10:427.

7. Jensen BC, Sivam D, Kifer CT, Myler PJ, Parsons M: Widespread variation in transcript abundance within and across developmental stages of Trypanosoma brucei. BMC Genomics 2009, 10:482.

8. Veitch NJ, Johnson PC, Trivedi U, Terry S, Wildridge D, MacLeod A: Digital gene expression analysis of two life cycle stages of the human-infective parasite, Trypanosoma brucei gambiense reveals differentially expressed clusters of co-regulated genes. BMC Genomics 2010, 11:124.

9. Siegel TN, Hekstra DR, Wang X, Dewell S, Cross GA: Genome-wide analysis of mRNA abundance in two life-cycle stages of Trypanosoma brucei and identification of splicing and polyadenylation sites. Nucleic Acids Res 2010, 38:4946-4957.

10. Kolev NG, Franklin JB, Carmi S, Shi H, Michaeli S, Tschudi C: The transcriptome of the human pathogen Trypanosoma brucei at single-nucleotide resolution. PLoS Pathog 2010, 6:e1001090.

11. Nilsson D, Gunasekera K, Mani J, Osteras M, Farinelli L, Baerlocher L, Roditi I, Ochsenreiter T: Spliced leader trapping reveals widespread alternative splicing patterns in the highly dynamic transcriptome of Trypanosoma brucei. PLoS Pathog 2010, 6:e1001037.

12. Queiroz R, Benz C, Fellenberg K, Hoheisel JD, Clayton C: Transcriptome analysis of differentiating trypanosomes reveals the existence of multiple post-transcriptional regulons. BMC Genomics 2009, 10:495

13. Clayton C, Shapira M: Post-transcriptional regulation of gene expression in trypanosomes and leishmanias. Mol Biochem Parasitol 2007, 156:93-101.

14. Berberof M, Vanhamme L, Tebabi P, Pays A, Jefferies D, Welburn S, Pays E: The 3'-terminal region of the mRNAs for VSG and procyclin can confer stage specificity to gene expression in Trypanosoma brucei. EMBO J 1995, 14:2925-2934

15. Furger A, Schürch $N$, Kurath $U$, Roditi I: Elements in the $3^{\prime}$ untranslated region of procyclin mRNA regulate expression in insect forms of Trypanosoma brucei by modulating RNA stability and translation. Mol Cell Biol 1997, 17:4372-4380.

16. Li J, Ruyechan WT, Williams N: Stage-specific translational efficiency and protein stability regulate the developmental expression of p37, an RNA binding protein from Trypanosoma brucei. Biochem Biophys Res Commun 2003, 306:918-923.

17. Gale MJ Jr, Carter V, Parsons M: Translational control mediates the developmental regulation of the Trypanosoma brucei Nrk protein kinase. J Biol Chem 1994, 269:31659-31665. 
18. Hotz HR, Hartmann C, Huober K, Hug M, Clayton C: Mechanisms of developmental regulation in Trypanosoma brucei: A polypyrimidine tract in the 3'-untranslated region of a surface protein mRNA affects RNA abundance and translation. Nucleic Acids Res 1997, 25:3017-3025.

19. Capewell P, Monk S, Ivens A, Macgregor P, Fenn K, Walrad P, Bringaud F, Smith TK, Matthews KR: Regulation of Trypanosoma brucei total and polysomal mRNA during development within its mammalian host. PLOS One 2013, 8:e67069.

20. Vasquez JJ, Hon CC, Vanselow JT, Schlosser A, Siegel TN: Comparative ribosome profiling reveals extensive translational complexity in different Trypanosoma brucei life cycle stages. Nucleic Acids Res 2014, 42:3623-3637.

21. Martinez-Calvillo S, Nguyen D, Stuart K, Myler PJ: Transcription initiation and termination on Leishmania major chromosome 3. Eukaryot Cell 2004, 3:506-517.

22. Myler PJ, Audleman L, deVos T, Hixson G, Kiser P, Lemley C, Magness C, Rickel E, Sisk E, Sunkin S, Swartzell S, Westlake T, Bastien P, Fu G, Ivens A, Stuart K: Leishmania major Friedlin chromosome 1 has an unusual distribution of protein-coding genes. Proc Natl Acad Sci U S A 1999, 96:2902-2906.

23. El Sayed NM, Myler PJ, Blandin G, Berriman M, Crabtree J, Aggarwal G, Caler E, Renauld H, Worthey EA, Hertz-Fowler C, Ghedin E, Peacock C, Bartholomeu DC, Haas BJ, Tran AN, Wortman JR, Alsmark UC, Angiuoli S, Anupama A, Badger J, Bringaud F, Cadag E, Carlton JM, Cerqueira GC, Creasy T, Delcher AL, Djikeng A, Embley TM, Hauser C, Ivens AC, et al: Comparative genomics of trypanosomatid parasitic protozoa. Science 2005, 309:404-409.

24. Liang XH, Haritan A, Uliel S, Michaeli S: Trans and cis splicing in trypanosomatids: mechanism, factors, and regulation. Eukaryot Cell 2003, 2:830-840.

25. Clayton CE: Networks of gene expression regulation in Trypanosoma brucei. Mol Biochem Parasitol 2014, 195:96-106.

26. Schwanhausser B, Busse D, Li N, Dittmar G, Schuchhardt J, Wolf J, Chen W, Selbach M: Global quantification of mammalian gene expression control. Nature 2011, 473:337-342.

27. Sonenberg $N$, Hinnebusch AG: Regulation of translation initiation in eukaryotes: mechanisms and biological targets. Cell 2009, 136:731-745.

28. Parsyan A, Svitkin Y, Shahbazian D, Gkogkas C, Lasko P, Merrick WC, Sonenberg N: mRNA helicases: the tacticians of translational control. Nat Rev Mol Cell Biol 2011, 12:235-245.

29. Villalba A, Coll O, Gebauer F: Cytoplasmic polyadenylation and translational control. Curr Opin Genet Dev 2011, 21:452-457.

30. Jackson RJ, Hellen CU, Pestova TV: The mechanism of eukaryotic translation initiation and principles of its regulation. Nat Rev Mol Cell Biol 2010, 11:113-127.

31. Hsieh AC, Liu Y, Edlind MP, Ingolia NT, Janes MR, Sher A, Shi EY, Stumpf CR, Christensen C, Bonham MJ, Wang S, Ren P, Martin M, Jessen K, Feldman ME, Weissman JS, Shokat KM, Rommel C, Ruggero D: The translational landscape of mTOR signalling steers cancer initiation and metastasis. Nature 2012, 485:55-61.

32. Joyce BR, Tampaki Z, Kim K, Wek RC, Sullivan WJ Jr: The unfolded protein response in the protozoan parasite Toxoplasma gondii features translational and transcriptional control. Eukaryot Cell 2013, 12:979-989.

33. Liu MJ, Wu SH, Wu JF, Lin WD, Wu YC, Tsai TY, Tsai HL, Wu SH: Translational landscape of photomorphogenic arabidopsis. Plant Cell 2013, 25:3699-3710.

34. Zhang M, Joyce BR, Sullivan WJ Jr, Nussenzweig V: Translational control in plasmodium and toxoplasma parasites. Eukaryot Cell 2012, 12:161-167.

35. Zhang M, Fennell C, Ranford-Cartwright L, Sakthivel R, Gueirard P, Meister S, Caspi A, Doerig C, Nussenzweig RS, Tuteja R, Sullivan WJ Jr, Roos DS, Fontoura BM, Menard R, Winzeler EA, Nussenzweig V: The Plasmodium eukaryotic initiation factor-2alpha kinase IK2 controls the latency of sporozoites in the mosquito salivary glands. J Exp Med 2010, 207:1465-1474.

36. Ingolia NT, Ghaemmaghami S, Newman JR, Weissman JS: Genome-wide analysis in vivo of translation with nucleotide resolution using ribosome profiling. Science 2009, 324:218-223.

37. Ingolia NT, Brar GA, Rouskin S, McGeachy AM, Weissman JS: The ribosome profiling strategy for monitoring translation in vivo by deep sequencing of ribosome-protected mRNA fragments. Nat Protoc 2012,

7:1534-1550.
38. Guttman M, Russell P, Ingolia NT, Weissman JS, Lander ES: Ribosome profiling provides evidence that large noncoding RNAs do not encode proteins. Cell 2013, 154:240-251.

39. Chew GL, Pauli A, Rinn JL, Regev A, Schier AF, Valen E: Ribosome profiling reveals resemblance between long non-coding RNAs and 5' leaders of coding RNAs. Development 2013, 140:2828-2834.

40. McCarthy DJ, Chen Y, Smyth GK: Differential expression analysis of multifactor RNA-Seq experiments with respect to biological variation. Nucleic Acids Res 2012, 40:4288-4297.

41. Mani J, Guttinger A, Schimanski B, Heller M, Acosta-Serrano A, Pescher P, Spath G, Roditi I: Alba-domain proteins of Trypanosoma brucei are cytoplasmic RNA-binding proteins that interact with the translation machinery. PLoS One 2011, 6:e22463.

42. Estevez AM: The RNA-binding protein TbDRBD3 regulates the stability of a specific subset of mRNAs in trypanosomes. Nucleic Acids Res 2008, 36:4573-4586

43. Droll D, Minia I, Fadda A, Singh A, Stewart M, Queiroz R, Clayton C: Post-transcriptional regulation of the trypanosome heat shock response by a zinc finger protein. PLoS Pathog 2013, 9:e1003286.

44. Ingolia NT, Brar GA, Stern-Ginossar N, Harris MS, Talhouarne GJ, Jackson SE, Wills MR, Weissman JS: Ribosome profiling reveals pervasive translation outside of annotated protein-coding genes. Cell Rep 2014, 8:1365-1379.

45. Kirstein-Miles J, Scior A, Deuerling E, Morimoto Rl: The nascent polypeptide-associated complex is a key regulator of proteostasis. EMBO J 2013, 32:1451-1468.

46. Lacombe T, Garcia-Gomez JJ, de La Cruz J, Roser D, Hurt E, Linder P, Kressler D: Linear ubiquitin fusion to Rps31 and its subsequent cleavage are required for the efficient production and functional integrity of $40 \mathrm{~S}$ ribosomal subunits. Mol Microbiol 2009, 72:69-84.

47. Bringaud F, Riviere L, Coustou V: Energy metabolism of trypanosomatids: adaptation to available carbon sources. Mol Biochem Parasitol 2006, 149:1-9.

48. Fairlamb AH, Opperdoes FR: Carbohydrate Metabolism In African Trypanosomes, With Special Reference To The Glycosome. In Carbohydrate Metabolism in Cultured Cells. Edited by Morgan MJ. New York Plenum Publishing Corp; 1986:183-224.

49. Anders S, Huber W: Differential expression analysis for sequence count data. Genome Biol 2010, 11:R106.

50. Roy $B$, Jacobson $A$ : The intimate relationships of mRNA decay and translation. Trends Genet 2013, 29:691-699.

51. Barbosa C, Peixeiro I, Romao L: Gene expression regulation by upstream open reading frames and human disease. PLOS Genet 2013, 9:e1003529.

52. Spriggs KA, Bushell M, Willis AE: Translational regulation of gene expression during conditions of cell stress. Mol Cell 2010, 40:228-237.

53. von Arnim AG, Jia Q, Vaughn JN: Regulation of plant translation by upstream open reading frames. Plant Sci 2014, 214:1-12.

54. Arribere JA, Gilbert WV: Roles for transcript leaders in translation and mRNA decay revealed by transcript leader sequencing. Genome Res 2013, 23:977-987

55. Berriman M, Ghedin E, Hertz-Fowler C, Blandin G, Renauld H, Bartholomeu DC, Lennard NJ, Caler E, Hamlin NE, Haas B, Bohme U, Hannick L, Aslett MA, Shallom J, Marcello L, Hou L, Wickstead B, Alsmark UC, Arrowsmith C, Atkin RJ, Barron AJ, Bringaud F, Brooks K, Carrington M, Cherevach I, Chillingworth TJ, Churcher C, Clark LN, Corton CH, Cronin A, et al: The genome of the African trypanosome Trypanosoma brucei. Science 2005, 309:416-422.

56. Butter F, Bucerius F, Michel M, Cicova Z, Mann M, Janzen CJ: Comparative proteomics of two life cycle stages of stable isotope-labeled Trypanosoma brucei reveals novel components of the parasite's host adaptation machinery. Mol Cell Proteomics 2013, 12:173-179.

57. Clayton CE: Most proteins, including fructose bisphosphate aldolase, are stable in the procyclic trypomastigote form of Trypanosoma brucei. Mol Biochem Parasitol 1988, 28:43-46.

58. Pichon X, Wilson LA, Stoneley M, Bastide A, King HA, Somers J, Willis AE: RNA binding protein/RNA element interactions and the control of translation. Curr Protein Pept Sci 2012, 13:294-304.

59. Hamilton TL, Stoneley M, Spriggs KA, Bushell M: TOPs and their regulation. Biochem Soc Trans 2006, 34:12-16.

60. Meyuhas O: Synthesis of the translational apparatus is regulated at the translational level. Eur J Biochem 2000, 267:6321-6330.

61. Jimenez-Lopez S, Mancera-Martinez E, Donayre-Torres A, Rangel C, Uribe L, March S, Jimenez-Sanchez G, de JE S: Expression profile of maize 
(Zea mays L.) embryonic axes during germination: translational regulation of ribosomal protein mRNAs. Plant Cell Physiol 2011 52:1719-1733

62. Bailey $\mathrm{TL}$, Elkan C: Fitting a mixture model by expectation maximization to discover motifs in biopolymers. Proc Int Conf Intell Syst Mol Biol 1994, 2:28-36.

63. Brecht M, Parsons M: Changes in polysome profiles accompany trypanosome development. Mol Biochem Parasitol 1998, 97:189-198.

64. Hirumi H, Hirumi K: Continuous cultivation of Trypanosoma brucei blood stream forms in a medium containing a low concentration of serum protein without feeder cells. J Parasitol 1989, 75:985-989.

65. Brun R, Schoenonberger M: Cultivation and in vitro cloning of procyclic culture forms of Trypanosoma brucei in a semi-defined medium. Acta Trop 1979, 36:289-292.

66. Ingolia NT, Lareau LF, Weissman JS: Ribosome profiling of mouse embryonic stem cells reveals the complexity and dynamics of mammalian proteomes. Cell 2011, 147:789-802.

67. Mittra B, Cortez M, Haydock A, Ramasamy G, Myler PJ, Andrews NW: Iron uptake controls the generation of Leishmania infective forms through regulation of ROS levels. J Exp Med 2013, 210:401-416.

68. Langmead B, Salzberg SL: Fast gapped-read alignment with Bowtie 2. Nat Methods 2012, 9:357-359.

69. Carver T, Harris SR, Berriman M, Parkhill J, McQuillan JA: Artemis: an integrated platform for visualization and analysis of high-throughput sequence-based experimental data. Bioinformatics 2012, 28:464-469.

70. Anders S, Pyl PT, Huber W: HTSeq - A Python framework to work with high-throughput sequencing data. Biorxiv 2014, doi:10.1101/002824.

71. Saeed Al, Sharov V, White J, Li J, Liang W, Bhagabati N, Braisted J, Klapa M, Currier T, Thiagarajan M, Sturn A, Snuffin M, Rezantsev A, Popov D, Ryltsov A, Kostukovich E, Borisovsky I, Liu Z, Vinsavich A, Trush V, Quackenbush J: TM4: a free, open-source system for microarray data management and analysis. Biotechniques 2003, 34:374-378.

72. Rice P, Longden I, Bleasby A: EMBOSS: the European Molecular Biology Open Software Suite. Trends Genet 2000, 16:276-277.

doi:10.1186/1471-2164-15-911

Cite this article as: Jensen et al:: Extensive stage-regulation of translation revealed by ribosome profiling of Trypanosoma brucei. BMC Genomics 2014 15:911.

\section{Submit your next manuscript to BioMed Central and take full advantage of:}

- Convenient online submission

- Thorough peer review

- No space constraints or color figure charges

- Immediate publication on acceptance

- Inclusion in PubMed, CAS, Scopus and Google Scholar

- Research which is freely available for redistribution 